\title{
An effective cell-penetrating antibody delivery platform
}

\author{
Andreas Herrmann, ${ }^{1,2}$ Toshikage Nagao, ${ }^{1}$ Chunyan Zhang, ${ }^{1}$ Christoph Lahtz, ${ }^{1,2}$ Yi-Jia Li, ${ }^{1}$ \\ Chanyu Yue, ${ }^{1,2}$ Ronja Mülfarth, ${ }^{1}$ and Hua $\mathrm{Yu}^{1}$ \\ 'Department of Immuno-Oncology, Beckman Research Institute at City of Hope Comprehensive Cancer Center, Duarte, \\ California, USA. ${ }^{2}$ LACell at Sorrento Therapeutics, San Diego, California, USA.
}

\begin{abstract}
Despite their well-recognized success in the clinic, antibodies generally do not penetrate cellular membranes to target intracellular molecules, many of which underlie incurable diseases. Here we show that covalently conjugating phosphorothioated DNA oligonucleotides to antibodies enabled their efficient cellular internalization. Antibody cell penetration was partially mediated by membrane potential alteration. Moreover, without an antigen to bind, intracellular levels of the modified antibodies underwent cellular clearance, which involved efflux and lysosomal degradation, enabling detection of intended intracellular molecules as tested in fibroblasts, tumor cells, and T cells. This target-dependent cellular retention of modified antibodies extended to in vivo studies. Both local and systemic administrations of low doses of modified antibodies effectively inhibited intracellular targets, such as transcription factors Myc, interferon regulatory factor 4, and tyrosine-protein kinase SRC, and expression of their downstream genes in tumors, resulting in tumor cell apoptosis and tumor growth inhibition. This simple modification enables the use of antibodies to detect and modulate intracellular molecules in both cultured living cells and in whole animals, forming the foundation for a new paradigm for antibody-based research, diagnostics, and therapeutics.
\end{abstract}

Conflict of interest: AH and HY own shares of LACell, a start-up company based on the intellectual properties of the cell-penetrating antibody technology, which was licensed from City of Hope Medical Center by Sorrento Therapeutics.

Copyright: () 2019, American Society for Clinical Investigation.

Submitted: January 15, 2019

Accepted: May 31, 2019

Published: July 25, 2019.

Reference information: JCI Insight. 2019;4(14):e127474. https://doi. org/10.1172/jici.insight.127474.

\section{Introduction}

Compared with small-molecule drugs, antibody-based therapy has many advantages. They include target specificity and high affinity, ease in identifying drug candidates, and serum stability (1-9). Today, over 40 antibodies have been clinically approved for therapeutic intervention, many of which show notable efficacy (10). However, unlike small-molecule drugs that are capable of inhibiting some intracellular targets, the clinically approved antibody therapies can target only extracellular molecules. Additionally, many critical intracellular targets for disease remain intractable by small-molecule inhibitors. It is therefore highly desirable to efficiently deliver antibodies intracellularly, to make challenging targets amenable to therapy. Over the years, multiple studies, primarily in cultured cells, have shown the feasibility of facilitating antibodies' cellular internalization (11-13). Furthermore, a number of studies have shown the potential of therapeutic benefits in vivo of a nuclear-penetrating lupus anti-DNA autoantibody (14-17). The anti-DNA autoantibody entered the cell nucleus and was shown to inhibit DNA repair and selectively kill certain cancer cells that are highly vulnerable to DNA damage (16). Another study showed that injecting antibodies against an intracellular protein, PRL-3, into tumors in vivo led to B cell-dependent antitumor effects (18). More recently, it has been reported that a sophisticatedly engineered antibody, modified at several parts of the antibody, was able to selectively locate tumor cells, enter tumor cell cytosol, escape endosomes, and directly target RAS mutants, leading to antitumor effects in xenograft tumor models (19). These pioneering studies illustrate that antibodies against intracellular targets can have desired biological consequences. Nevertheless, a platform that enables antibody cellular/nuclear penetration to target virtually any intracellular molecule with 1 simple modification remains unavailable.

Our laboratory has identified many roles of STAT3 in promoting cancer (20-22). As a transcription factor, STAT3 proves a challenging target for small-molecule direct/specific inhibitors $(20,21,23)$. Our initial studies demonstrating successful trapping of STAT3 in cytoplasm by transfecting antibodies against STAT3 and its interacting protein, exportin 7 (our unpublished observations), led us hypothesize that efficient delivery of antibodies intracellularly could create effective drugs against transcription factors, such as STAT3. Parallel 
to the results of STAT3 cytoplasm trapping by antibodies, we unexpectedly observed that phosphorothioated (PS) single-stranded DNA (ssDNA) oligonucleotides (oligos) entered cells in a sequence-independent manner. These apparently unrelated observations prompted us to test whether attaching such DNA oligos to antibodies might enable antibody cell penetration and target recognition/inhibition. In the current study, using an efficient covalent attachment approach that has the potential for clinical development, we demonstrate that conjugating antibodies with PS ssDNA oligos enables them to penetrate cells, where they bind to the intended intracellular target antigens in live fibroblasts, tumor cells, and T cells. Furthermore, we demonstrate the feasibility of generating specific antibody-based inhibitors against intracellular targets, including transcription factors, such as interferon regulatory factor 4 (IRF4), Myc, and tyrosine-protein kinase SRC, leading to potent antitumor effects in various tumor models.

\section{Results}

Characterization of PS ssDNA oligo-modified targeting and nontargeting antibodies. Enabling antibodies to efficiently penetrate cellular membranes to detect and to modulate intracellular antigens/targets is highly desirable for not only research but also diagnostics and therapeutics. We covalently attached fluorescently labeled (FAM-labeled) PS or non-phosphorothioated (PO) DNA oligonucleotides to control IgG antibodies, tubulin antibodies, as well as IRF4, pY(418)-Src, and Myc antibodies. The DNA oligo-modified antibodies underwent high-performance liquid chromatography (HPLC) purification and electrophoresis to ensure the separation of the modified antibodies from unconjugated antibodies and oligos, as well as other undesired by-products or contaminants (Supplemental Figures 1 and 2; supplemental material available online with this article; https://doi.org/10.1172/jci.insight.127474DS1). We then tested the PS DNA oligo-modified antibodies for their ability to penetrate living cells and recognize their intended antigen(s). Compared with unmodified antibodies, covalent conjugation of PS DNA oligos to IRF4 and cMyc antibodies enabled efficient cell penetration, in a time- and dose-dependent manner (Supplemental Figure 3, A and B). Attaching PO DNA oligos to IRF4 and Myc antibodies or mixing equimolar or excess molar of PS DNA oligos with antibodies in the absence of conjugation did not permit internalization of the antibodies (Supplemental Figure 3C). Moreover, cellular uptake of the PS DNA oligo-modified antibodies was independent of the nucleic acid sequence phosphorothioated in the sugar-phosphate backbone (Supplemental Figure 3C).

To examine whether cellular internalization of PS DNA oligo-modified antibodies is dependent on PS DNA oligos, we performed competition assays between PS ssDNA oligos alone and PS DNA oligo-modified Myc antibodies. Our results indicated that PS ssDNA oligos could impede the entry of the PS DNA oligo-modified antibodies into cells in a dose-dependent manner (Supplemental Figure 3D). Furthermore, cellular uptake was not likely influenced significantly by $\mathrm{Fc}_{\mathrm{c}-} \gamma$ receptor $(\mathrm{Fc} \gamma \mathrm{R}$, Supplemental Figure $3 \mathrm{E})$, endocytic activity of tumor cells (Supplemental Figure 3F), or EGFR expression (Supplemental Figure 3 , $\mathrm{G}$ and $\mathrm{H}$ ). EGFR has been shown to mediate cellular uptake of PS modified antisense oligonucleotides (24), although PS modified CpG DNA oligos and CpG-linked conjugates enter immune cells $(25,26)$, which do not usually exhibit elevated expression of EGFR.

Because endosomal trapping is a major problem facing macromolecule intracellular delivery, we set out to identify the cellular location of the internalized PS DNA oligo-modified antibody. We cultured human A2058 melanoma cells with PS DNA oligo-modified (FAM-labeled) tubulin antibodies (green), followed by fixation and staining with another tubulin antibody (red) recognizing a different epitope. Confocal microscopy analyses showed that the PS DNA oligo-modified antitubulin antibody penetrated the outer cell membrane and colocalized with the tubulin cytoskeletal fiber network throughout the entire cytoplasm (Figure 1A and Supplemental Figures 4 and 5). Moreover, the ability of the PS DNA oligo-modified antitubulin antibody, but not its nontargeting antibody counterpart, to recognize its intracellular antigen in living cells was shown by an adapted immunoprecipitation assay — incubating live cells with modified antibodies — followed by lysate preparation for Western blotting, which we named Lilith-immunoprecipitation, LIP (Figure 1B). In this experiment, whole-cell lysis was performed immediately after antibody incubation in cultured living cells, allowing the detection of IgG heavy and light chains of the modified antibodies (Figure 1B).

Because blocking potential mediators of protein cellular entry by inhibitors and lack of expression of EGFR did not show significant effects on cell penetration of the modified antibodies (Supplemental Figure 3, E-H), we tested the possibility that changes in membrane potential may facilitate the entry of the PS DNA oligo-modified antibodies. It is appreciated that cells exhibit a low but increasing membrane potential during $G_{1}$, and membrane potential sharply rises once cells enter the $S$ phase. 
A

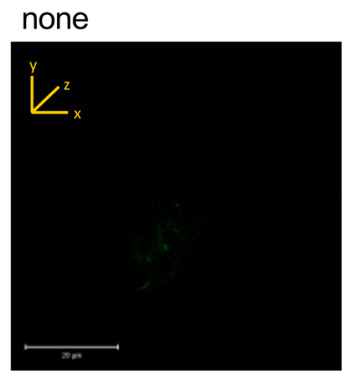

mod. $\alpha$ Tubulin
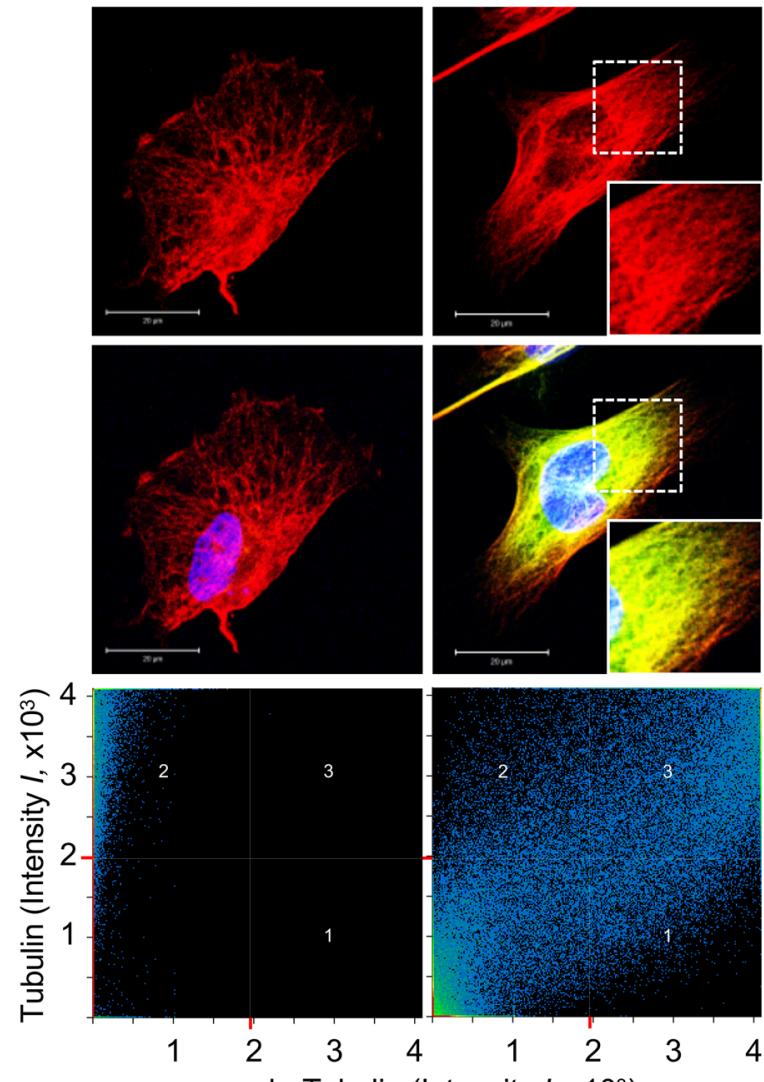

amod. $\alpha$ Tubulin

-Tubulin

口 Hoechst33342

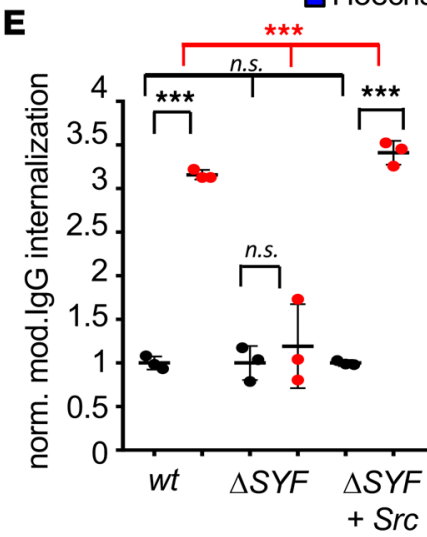

B

LIP:
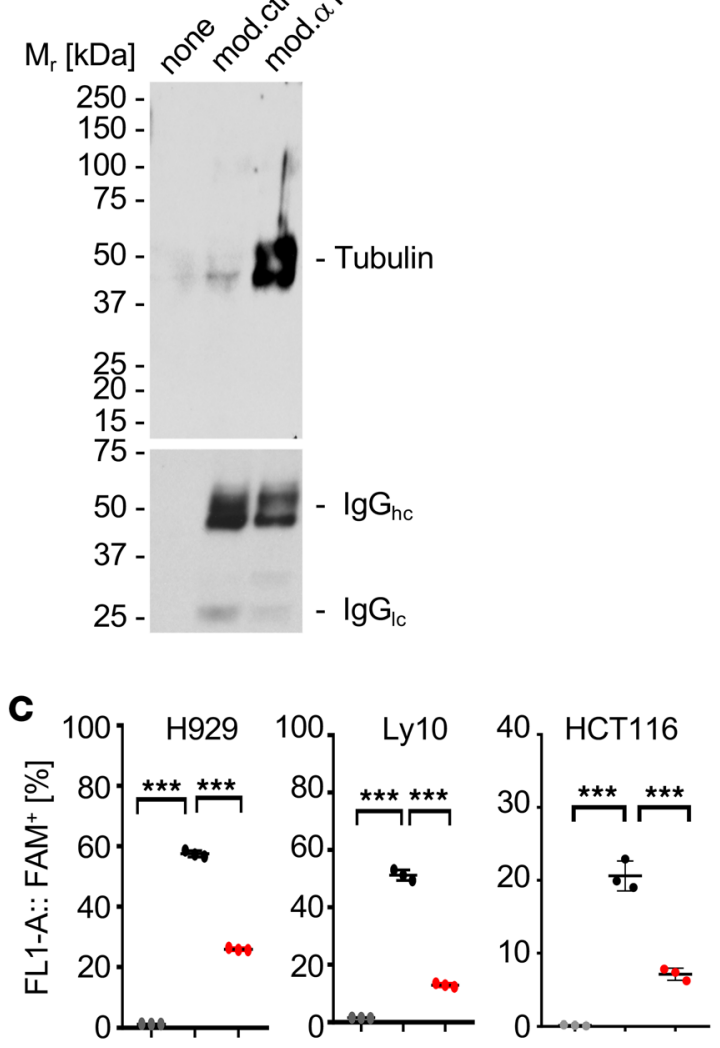

$\square$ blank control

mod.aTubulin

$\square$ mod. $\alpha$ Tubulin $+\mathrm{KCl}$

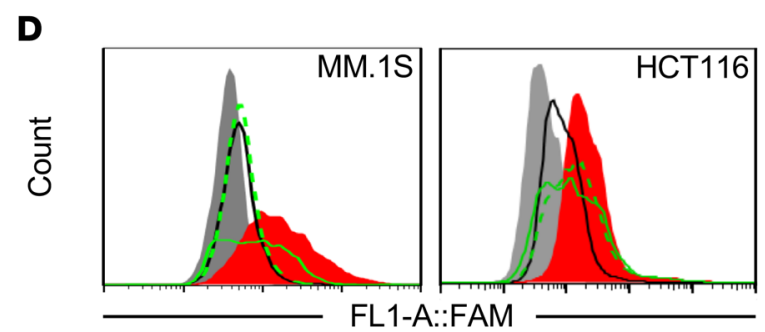

$\square$ blank control amod.IgG control $\square$ mod. $\alpha$ Tubulin IImod.IgG/iLYSO $\square$ mod.IgG/iEFFLUX 
Figure 1. PS DNA oligo-modified antibody cell penetration and antigen-dependent intracellular retention. (A) Representative 3D confocal microscopy with quantification showing binding of PS DNA oligo-modified tubulin antibody (green) to tubulin in living cells, which were fixed and stained by another tubulin antibody (red). Scale bar: $20 \mu \mathrm{m}$. Enlarged images are further magnified $\times 1.6$. (B) Western blotting detected tubulin from cell lysates prepared from cells cultured with the modified tubulin antibody. hc, heavy chain; Ic, light chain. (C) Membrane potential depolarization reduces cellular uptake of PS DNA oligomodified antibody assessed by flow cytometry performed in triplicate. (D) Representative flow cytometry assessing cellular clearance of PS DNA oligo-modified nontargeting antibody. (E) Flow cytometry indicates cellular retention of PS DNA oligo-modified antibody requires antigen. Data shown represent 1 of 4 independent experiments. SD shown. Unpaired Student's $t$ test (C and E) or 1-way ANOVA with Tukey's test (E). n.s., not significant, and ${ }^{* * *} P<0.001$.

The higher membrane potential is maintained throughout $S$ and $G_{2}$ (27). Furthermore, membrane potentials in an unsynchronized culture were similar to that of $S$ and $G_{2}$ in isolated synchronized cells (27). We tested cellular entry of the PS DNA oligo-modified antibodies, compared with unmodified or PO DNA oligo-modified antibodies, in unsynchronized human multiple myeloma MM.1S cells during different phases. Results from the experiment indicate that the PS DNA-modified antibodies entered efficiently into cells in $\mathrm{S}$ and $\mathrm{G}_{2}$ phase, especially cyclin A-positive cells (Supplemental Figure 3, I and J), compared with the pooled cells (Supplemental Figure 3, A-D). These data suggest that cellular internalization of the modified antibody is affected by membrane potential. To test whether reducing membrane potential could abrogate cellular internalization of the PS DNA oligo-modified antibodies, we induced membrane depolarization with potassium chloride $(\mathrm{KCl})$ in cultured cells. Our results indicate that membrane depolarization significantly reduced the modified antibody uptake in a variety of human cancer cell lines (Figure 1C).

To investigate the cytologic fate of modified antibodies in the absence of an antigen, we tested cellular retention of nontargeting modified IgG, under conditions allowing cellular clearance (see Methods). Although modified nontargeting IgG did not undergo efficient cellular accumulation compared with modified targeting antibodies (Supplemental Figure 6), blocking cellular efflux using verapamil hydrochloride (iEFFLUX) increased the intracellular presence of nontargeting modified IgG in both human myeloma MM.1S cells and human colon cancer HCT116 tumor cells (Figure 1D). In addition, inhibiting lysosomal degradation using chloroquine (iLYSO) also allowed the detection of the modified nontargeting antibody inside HCT116 cells (Figure 1D), indicating that both processes could contribute to cellular clearance of the modified nontargeting IgG antibodies, in addition to other unidentified mechanisms. These findings also imply that the binding of the modified targeting antibody to its target antigen may prevent its efflux or lysosomal degradation, enabling its cellular retention.

To determine whether the presence of intracellular target expression is required for the retention of the modified antibodies, we used a set of genetically engineered mouse embryonic fibroblast (MEF) cell lines, including MEF-WT, MEF- $\triangle$ SYF (Src kinases Src, Yes, Fyn deleted), as well as MEF- $\triangle$ SYF cells with reconstituted Src expression (MEF- $\Delta S Y F+S r c)$. Flow cytometric analyses of the MEF cell variants treated with the indicated modified antibodies, followed by time to allow cellular clearance of nontargeting modified antibodies, showed intracellular retention of modified pY(418)-Src antibody exclusively in cells with Src antigen expression (Figure 1E).

PS DNA oligo-modified antibody identifies live, IFN- $\gamma^{+}$T cells. A fast and simple method to isolate distinct live immune cell populations, such as cytokine-producing $\mathrm{T}$ cells, in an effort to perform mechanistic studies and to monitor immune responses, and to expand the activating or suppressing $\mathrm{T}$ cells for therapeutic purposes, is highly desirable yet remains elusive. We tested the potential of the modified, cell-penetrating IFN- $\gamma$ antibodies to monitor IFN- $\gamma$-producing, living splenic $\mathrm{CD}^{+} \mathrm{T}$ cells. As shown by flow cytometric analyses, FAM-labeled, modified, cell-penetrating IFN- $\gamma$ antibodies were retained in $\mathrm{CD}^{+} \mathrm{T}$ cells stimulated by PMA and ionomycin to produce IFN- $\gamma$. In contrast, the modified IgG control antibody was not detectable, under the same stimulation condition as the T cells treated with the modified anti-IFN- $\gamma$ antibodies (Figure $2 \mathrm{~A}$ ). To validate the IFN- $\gamma^{+} \mathrm{CD} 8^{+} \mathrm{T}$ cell population detected by the cell-penetrating IFN- $\gamma$ antibodies, we repeated incubation of living splenic $\mathrm{CD} 8^{+} \mathrm{T}$ cells with FAM-labeled, modified, cell-penetrating IFN- $\gamma$ antibodies with and without stimulation, this time followed by fixation and permeabilization of the cells before IFN- $\gamma$ restaining using an unmodified IFN- $\gamma$ antibody that recognizes a different epitope. The second IFN- $\gamma$ antibody using the conventional intracellular staining method detected the same IFN- $\gamma^{+}$ $\mathrm{CD}^{+} \mathrm{T}$ cell population as the modified IFN- $\gamma$ antibody, illustrated by the IFN- $\gamma$-double positive CD8 ${ }^{+} \mathrm{T}$ cell population (Figure $2 \mathrm{~B}$ ). These results suggest the ability of the cell-penetrating antibody technology to detect accurately live, cytokine-producing immune cells. 
A

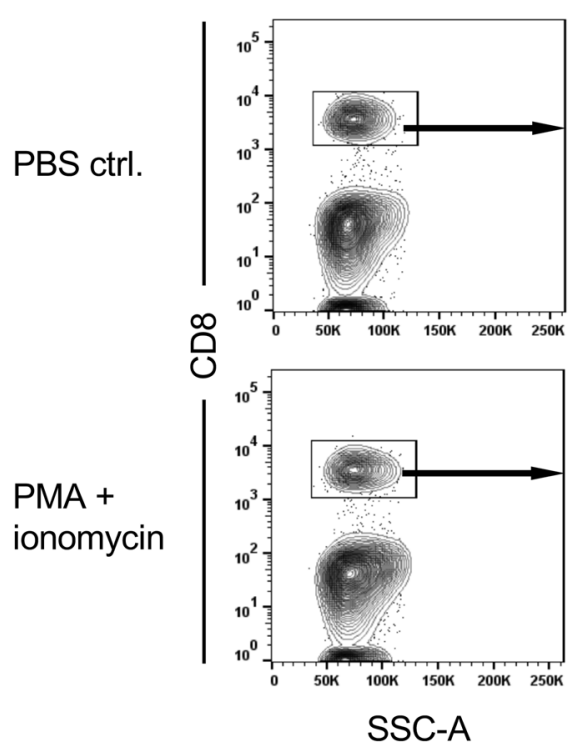

PBS ctrl.

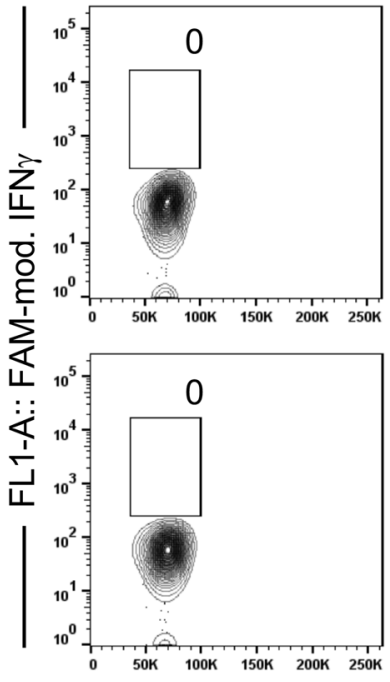

SSC-A
mod.lgGFAM ctrl.
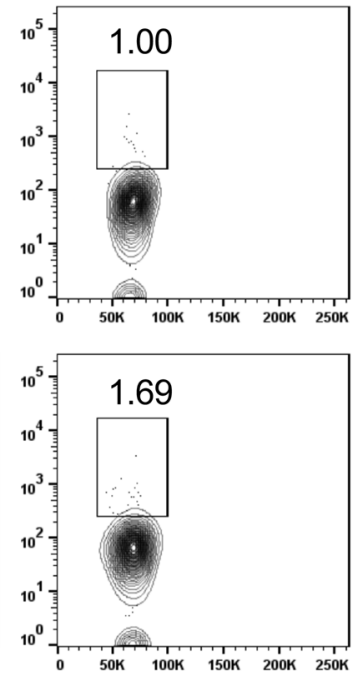

FSC-A

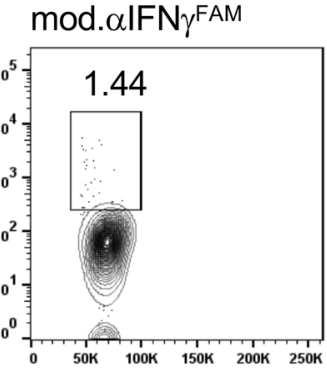

B
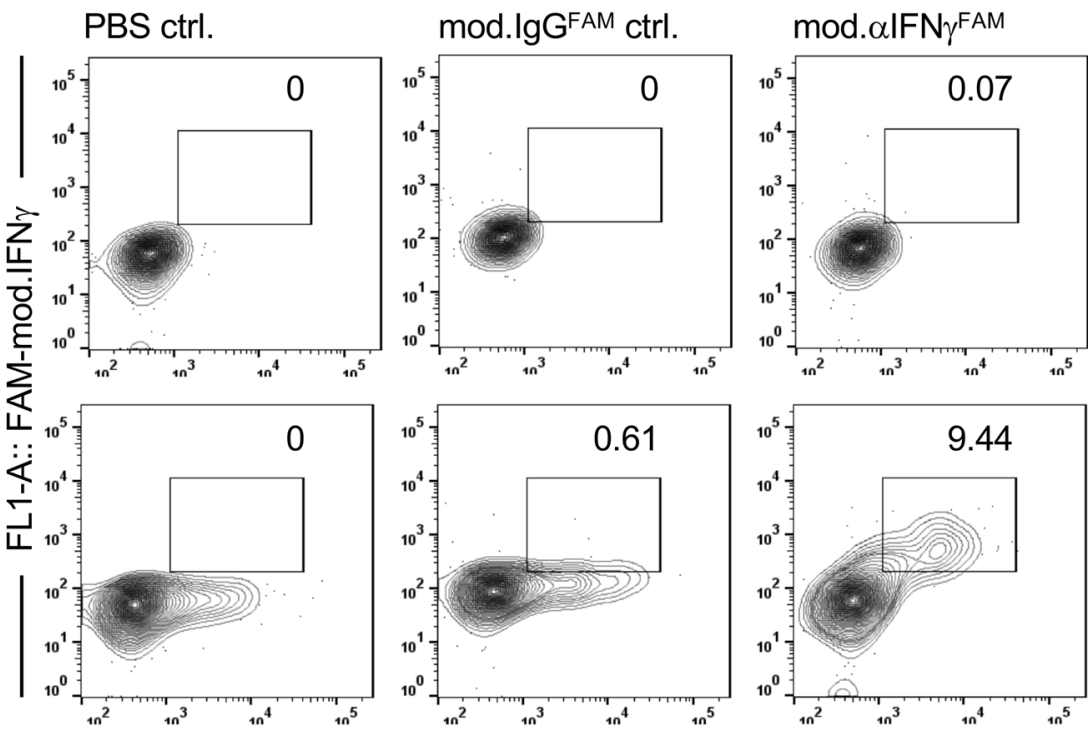

PBS ctrl.

PMA + ionomycin

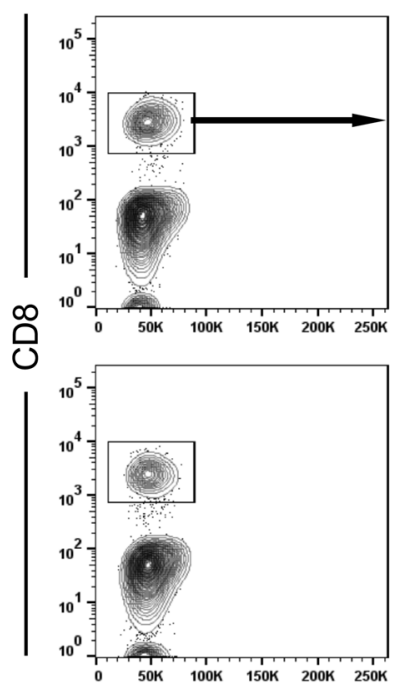

SSC-A
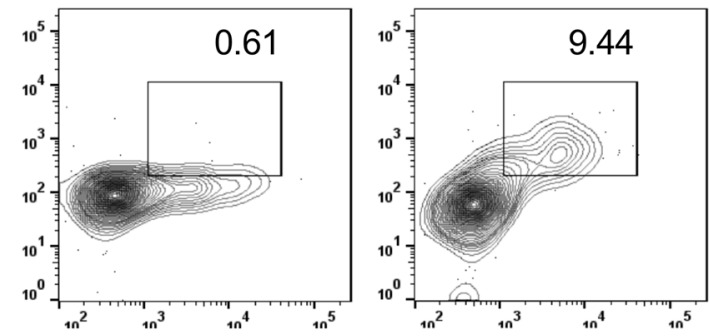

FL2-A:: PE-IFN

Figure 2. PS DNA oligo-modified antibody detects IFN- $\gamma$-producing, live $\mathbf{C D 8}^{+} \mathbf{T}$ cells. (A) Flow cytometry of mouse splenocytes cultured with indicated modified antibodies with or without stimulation for cytokine production. (B) IFN- $\gamma$-expressing live CD8 ${ }^{+}$T cells detected by FAM-labeled, PS DNA oligomodified IFN- $\gamma$ antibody was validated by subsequent cellular permeabilization and IFN- $\gamma$ intracellular staining using a phycoerythrin-labeled (PE-labeled) IFN- $\gamma$ antibody recognizing another IFN- $\gamma$ epitope. Data shown represent 1 of 3 experiments.

An IRF4 antibody-based inhibitor. Pioneering studies using a loss-of-function- and RNA interferencebased genetic screen have demonstrated that IRF4 is critical for tumor cell survival in myeloma (28-30). IRF4 triggers Myc oncoprotein expression, which in turn serves as a transcriptional enhancer for IRF4 expression (29). These findings led us to test cell-penetrating antibodies recognizing IRF4 for their ability to inhibit IRF4 in human multiple myeloma cells.

PS DNA oligo-modified anti-IRF4 antibody (which is a mouse IgG labeled with FAM DNA oligo) readily penetrated MM.1S cells and colocalized with IRF4 protein in the cell nucleus (Figure 3A). In addition to FAM DNA oligo, which was used for modifying the antibodies, anti-mouse IgG and anti-IRF4 antibodies were used for the immunostaining/microscopy analyses (Figure 3A). Intracellular accumulation of PS 
DNA oligo-modified anti-IRF4 antibody was rapid and robust as assessed by flow cytometry (Figure 3B). LIP Western blotting assay using cell lysates prepared from MM.1S cells cultured with indicated modified antibodies showed that PS DNA oligo-modified anti-IRF4 antibody bound to its intracellular target, IRF4 protein, in vitro and colocalized with target IRF4 protein in vivo (Figure 3C and Supplemental Figure 7). Colocalization of modified anti-Myc antibody with its target in tumor tissues after systemic treatments was also shown (Supplemental Figure 7). These data suggest that the PS ssDNA oligo modification of antibodies not only enables cellular internalization but also retains antigen recognition specificity in vitro and in vivo.

Treating MM.1S cells in vitro with the PS DNA oligo-modified anti-IRF4 antibody resulted in significantly reduced expression of IRF4 and MYC at both mRNA and protein levels, whereas the modified control IgG did not (Figure 3D). Moreover, the potential of PS DNA oligo-modified antiIRF4 antibody to increase tumor cell apoptosis was shown by elevated cleaved poly (ADP-ribose) polymerase (PARP) expression (Figure 3D). The results suggest that treating myeloma cells with PS DNA oligo-modified anti-IRF4 antibody not only disrupted intracellular IRF4 signaling but also resulted in antitumor activity in vitro (Supplemental Figure 8). Similarly, PS DNA oligo-modified anti-Myc antibody impedes Myc DNA binding and significantly reduces tumor cell proliferation of Myc-dependent MDA-MB-231 but not of Myc-independent MCF7 breast cancer cells (ref. 31; Supplemental Figure 8, E and F). Furthermore, PS ssDNA oligo-modified Myc antibody treatment significantly regulated target gene expression. It reduced expression of prosurvival/proliferating genes $B C L 2$ and CCND2 but increased expression of GADD45A and $C D K N 2 B$, which are involved in cell growth/cell cycle arrest (Supplemental Figure 8G). A significantly increased sub- $\mathrm{G}_{1}$ apoptotic cell population was also detected following long-term treatment of MM.1S tumor cells by the PS DNA oligo-modified anti-IRF4 antibody (Figure 3E and Supplemental Figure 9).

Antitumor effects of an IRF4 antibody inhibitor. Lenalidomide and Bruton's tyrosine kinase (BTK) inhibitor toxicity against activated B cell-like (ABC) diffuse large B cell lymphoma is in part induced by inhibiting IRF4 $(28,32,33)$. Although combinatory antitumor effects of the 2 inhibitors are highly significant, either inhibitor had only moderate inhibitory effects in vivo on a subcutaneous ABC lymphoma in a mouse tumor model (33). To date, a direct IRF4 inhibitor is not available to test IRF4 as a target for myeloma and ABC lymphoma treatment in vivo. Treating mice bearing human multiple myeloma MM.1S tumors by a low dose $(10 \mu \mathrm{g}$ per treatment administered locally) of the cell-penetrating anti-IRF4 antibodies resulted in significantly delayed tumor growth kinetics (Figure 4A). Immunohistochemical analyses of the treated tumors also indicated that treatment with PS DNA oligo-modified anti-IRF4 antibody led to loss of tumor tissue integrity, significantly decreased proliferative activity, and induced tumor vasculature collapse, which was quantified (Figure 4A). A reduction of IRF4 nuclear accumulation in the modified IRF4-treated tumors was also detectable (Figure 4B).

Antitumor effects by systemic treatments with modified IRF4 or Myc antibodies. Systemic treatments with PS DNA oligo-modified anti-IRF4 or anti-Myc antibody (10 $\mu \mathrm{g} /$ treatment) of s.c. engrafted human MM.1S multiple myeloma cells led to robust and significantly decreased tumor growth kinetics (Figure 4C). By assessing the DNA-binding activity of the 2 transcription factors using DNA oligo pull-down assays, we showed that both IRF4 and Myc underwent considerably reduced DNA-binding activity in tumors upon systemic treatments with their respective PS DNA oligo-modified antibodies, compared with the modified IgG control antibody or vehicle control (Figure 4D). Tumor tissue section histology analysis indicated disruption of tissue integrity upon systemic treatments with modified anti-IRF4 or anti-Myc antibody, which was accompanied by significantly decreased CD $31^{+}$tumor vasculature and $\mathrm{Ki}^{+} 7^{+}$proliferative activity (Figure 4, E and F). Furthermore, RT-PCR analyses indicated significantly reduced mRNA expression of IRF4 and Myc downstream genes, such as PRDM1 and CAD (Figure 4G), indicating systemic treatments with PS DNA oligo-modified anti-IRF4 or anti-Myc antibody suspended intracellular signaling of IRF4 and Myc. Moreover, antibody targeting of transcription factors IRF4 or Myc significantly reduced mRNA expression of key molecules driving tumor cell cycle progression (CDK4, CDK6, CCND1; Figure 4H). Systemic treatments with PS DNA oligo-modified anti-IRF4 or anti-Myc antibody also significantly elevated the mRNA expression of critical caspases CASP3, CASP8, and $C A S P 9$, which are involved in inducing tumor cell death (Figure 4I).

A $p Y$ (418)-Src antibody inhibitor. The nonreceptor Src tyrosine kinase resides at the inner side of the cell membrane, anchored by its N-terminal SH4 domain. Unlike viral Src (v-Src) encoded by the Rous sarcoma virus, cellular Src contains a regulatory tyrosine, Y527, considered inhibitory toward signal-ac- 
A

none

mod. $\alpha$ IRF-4FAM

unmod. $\alpha$ IRF-4

B

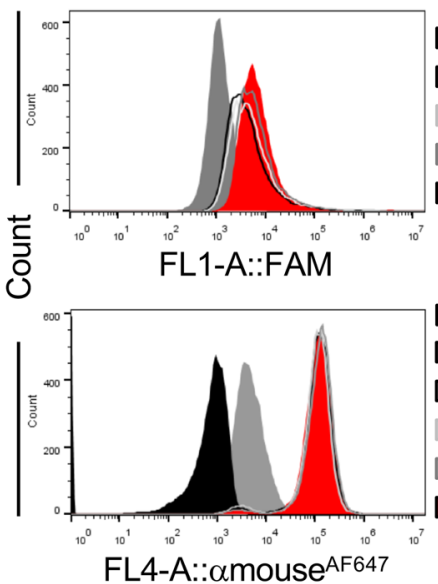

$\square$ BLANK

$\square 0.5 \mathrm{~h}$ mod. $\alpha \mathrm{IRF}-4$

$\square 1 \mathrm{~h}$ mod. $\alpha \mathrm{IRF}-4$

$\square 2 \mathrm{~h}$ mod. $\alpha \mathrm{IRF}-4$

$\square 4 \mathrm{~h}$ mod. $\alpha \mathrm{IRF}-4$

D

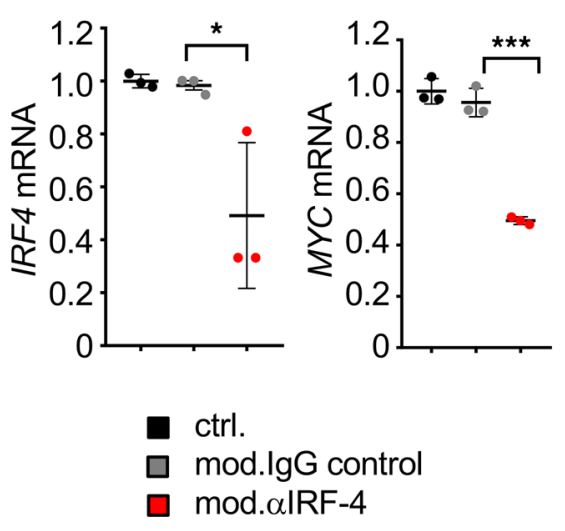

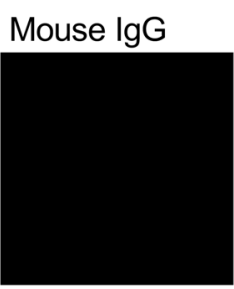
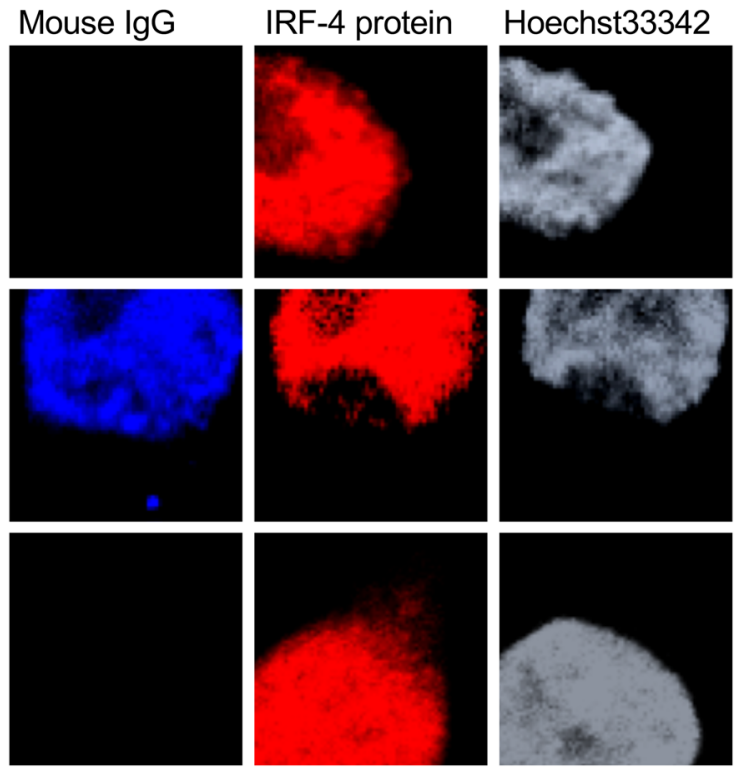

C

LIP:

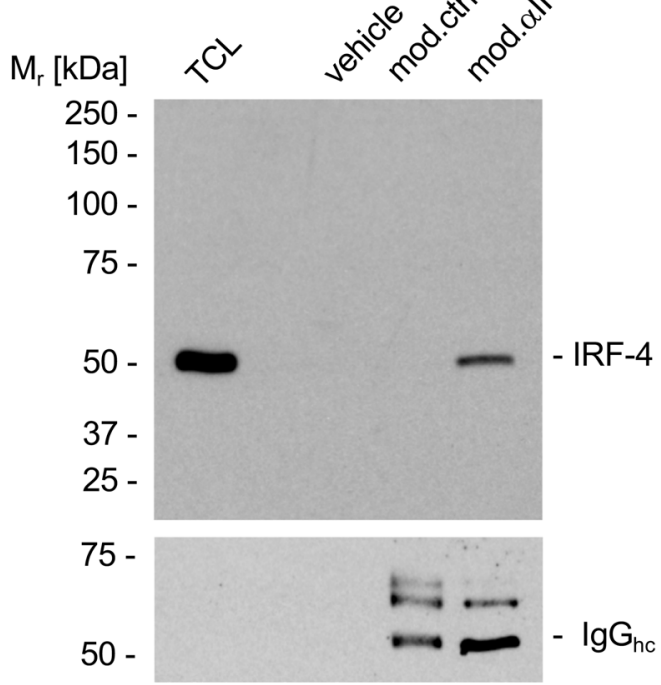

E

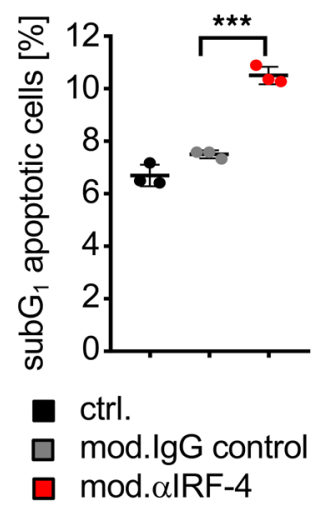


Figure 3. PS DNA oligo-modified IRF4 antibody inhibits IRF4. (A) Representative FAM signals and mouse IgC or IRF4 protein staining showing PS DNA oligo-modified IRF4 antibodies' colocalization with IRF4. Scale bar: $20 \mu \mathrm{m}$. Insets are further magnified $\times 7.5$. (B) Kinetics of cell entry of the modified antibodies detected by FAM and anti-mouse IgG antibody. (C) IRF4 recognition by PS DNA oligo-modified antibodies in living cells shown by representative Western blotting from lysates prepared from cells cultured with the indicated modified antibodies. TCL, total cell lysate (D) Representative real-time PCR (RT-PCR) and Western blotting showing effects of the modified IRF4 antibody on expression of IRF4 and its target genes performed in triplicate. (E) Flow cytometry detecting apoptotic cells (sub- $G_{1}$ population) by the PS DNA oligo-modified antibodies performed in triplicate. SD shown. Unpaired Student's $t$ test: ${ }^{*} P<0.05$, and ${ }^{* * *} P<0.001$.

tivating Y418. Although v-Src is truncated at 526aa, Src tyrosine kinase has been found to be overexpressed and highly activated at Y418 in numerous human cancers $(34,35)$. Phosphorylated tyrosine 418 of Src [pY(418)-Src] is therefore considered a desirable target for cancer therapy, including for human breast carcinoma, colon carcinoma, pancreatic cancer, and melanoma (36).

We first assessed whether the PS DNA oligo-modified anti-pY(418)-Src antibody retained its ability to recognize its antigen (Supplemental Figure 10), followed by assessing whether it efficiently penetrated live cells and homed to where its intracellular target resides (Figure 5A). We then performed colocalization studies using human melanoma A2058 cells, which require Src activation for growth/survival (37-39). As shown by confocal microscopy, PS DNA oligo-modified pY(418)-Src antibodies colocalized with pY(418)-Src at the inner side of cell membrane (Figure 5B). PS DNA oligo-modified pY(418)-Src antibodies underwent cellular internalization robustly as demonstrated by cellular uptake kinetics analyzed by flow cytometry (Figure 5C). Using LIP assay, we further showed that the PS DNA oligo-modified pY(418)-Src antibody recognized and precipitated its target, pY(418)-Src, in living tumor cells (Figure 5D). Importantly, the PS DNA oligo-modified pY(418)-Src antibody was detectable in the treated tumor tissues, whereas the modified nontargeting IgG control antibody was not (Figure 5E). These results suggest that only the PS DNA oligo-modified nontargeting IgG control antibody, but not the modified targeting antibody, underwent tissue clearance, within 24 hours after the last treatment. Cellular and tissue clearance of the PS DNA oligo-modified nontargeting IgG control antibody or surplus antibody might also be due to cellular efflux and lysosomal degradation, as shown in cultured cells (Figure 1D).

Antitumor effects of PS DNA oligo-modified pY(418)-Src antibody. Treating human melanoma A2058 tumor-bearing mice with the PS DNA oligo-modified pY(418)-Src antibody (10 $\mu \mathrm{g} /$ treatment) significantly decreased tumor growth kinetics compared with the modified nontargeting IgG control antibody (Figure 6A). PS DNA oligo modification of pY(418)-Src antibody did not interfere with antigen recognition and function (Figure 6B and Supplemental Figure 10). Treating the human melanoma tumors locally with modified anti-pY(418)-Src resulted in an increase in protein expression of tumor suppressor p53, which is known to be inhibited by activated STAT3 (40), as well as cleaved caspase-3, as shown by Western blot analyzing tumor tissue homogenates (Figure 6C). Immunohistochemical analyses of the tumor tissue sections also indicated that administration of PS DNA oligo-modified cell-penetrating pY(418)-Src antibody led to considerable loss of tumor tissue integrity and significantly reduced $\mathrm{CD} 31^{+}$tumor vasculature and diminished Src kinase activity (Figure 6D). Moreover, mRNA expression of cell cycle inhibitors $C D K N 2 A$ and $C D K N 2 B$ was significantly elevated while expression of matrix metalloproteinase $M M P 1$, involved in tumor cell metastasis, and tumor cell survival factor survivin (BIRC5) was significantly decreased upon treatment with PS DNA oligo-modified pY(418)-Src antibody (Figure 6E). Hence, PS DNA oligo-modified pY(418)Src antibody represents a promising, highly specific Src kinase inhibitor.

\section{Discussion}

Our studies demonstrate that covalently linking antibodies with PS DNA oligos, regardless of the oligo sequences, enables antibodies to penetrate cell membranes, escape endosomal degradation, recognize intracellular target antigens, and exert target-specific antitumor effects. Our cell-penetrating antibody approach originated from several unrelated studies and unexpected observations, which were followed by designed tests involving noncovalent and various covalent attachment chemistries.

Although endosomal trapping has been a major challenge for intracellular functional delivery of RNA, peptides, and antibodies, our confocal microscopic studies with the PS DNA oligo-modified tubulin antibodies show that the delivered antibodies coincide with the tubulin structure. The majority of the current antibody intracellular delivery methodologies are unidirectional (11-19). This study shows that cellular retention of our modified antibodies requires the presence of a target antigen because the modified nontargeting antibodies, within a relatively short time, become absent in cultured living cells and in treated 
A

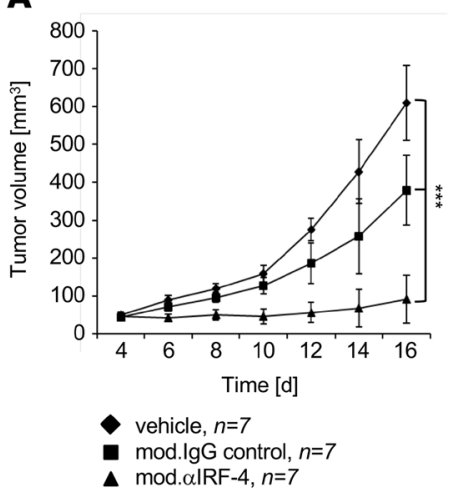

B
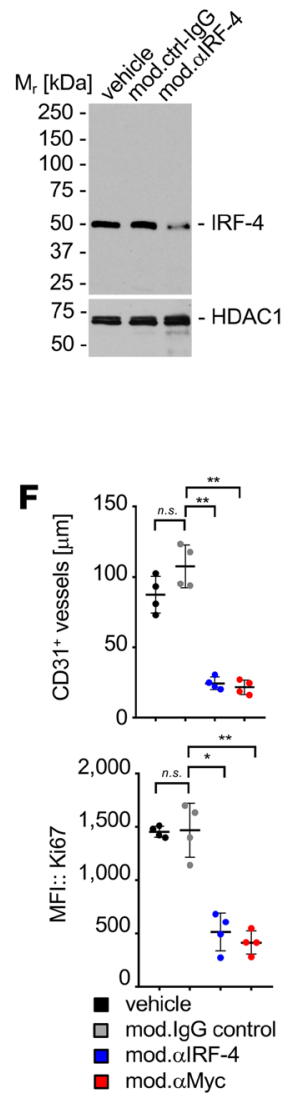

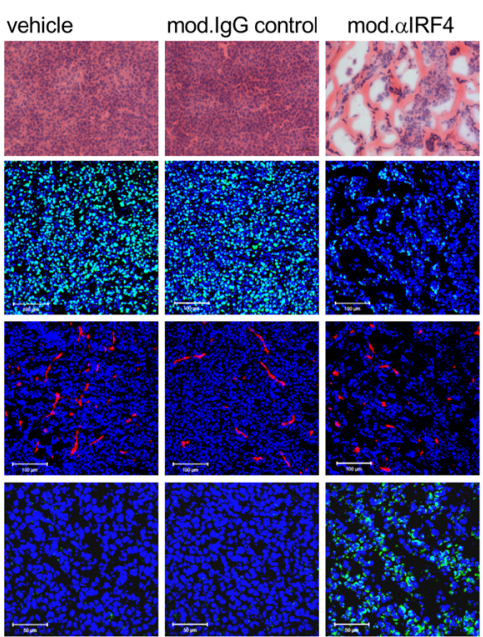

$\square / \square \mathrm{H \& E}$

ㅁi67

- Hoechst33342

CD31

- Hoechst33342

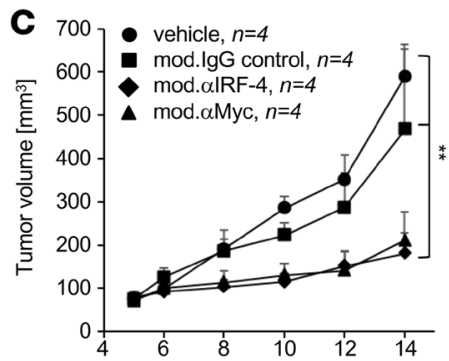

口cl.Caspase 3 - Hoechst33342
D
oligo pull-down

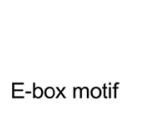

IRF4 binding motif

INPUT

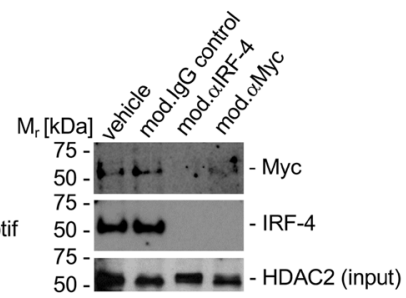

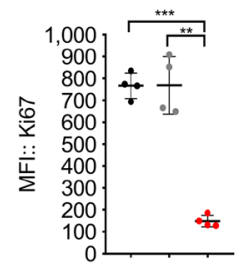
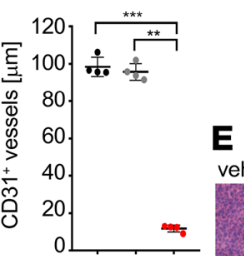

Time [d]

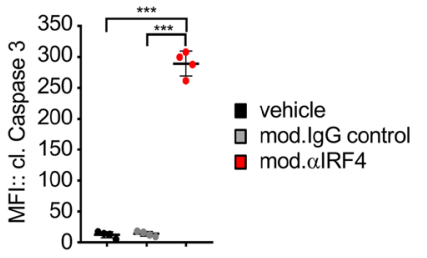

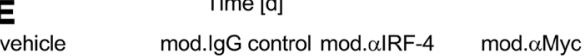
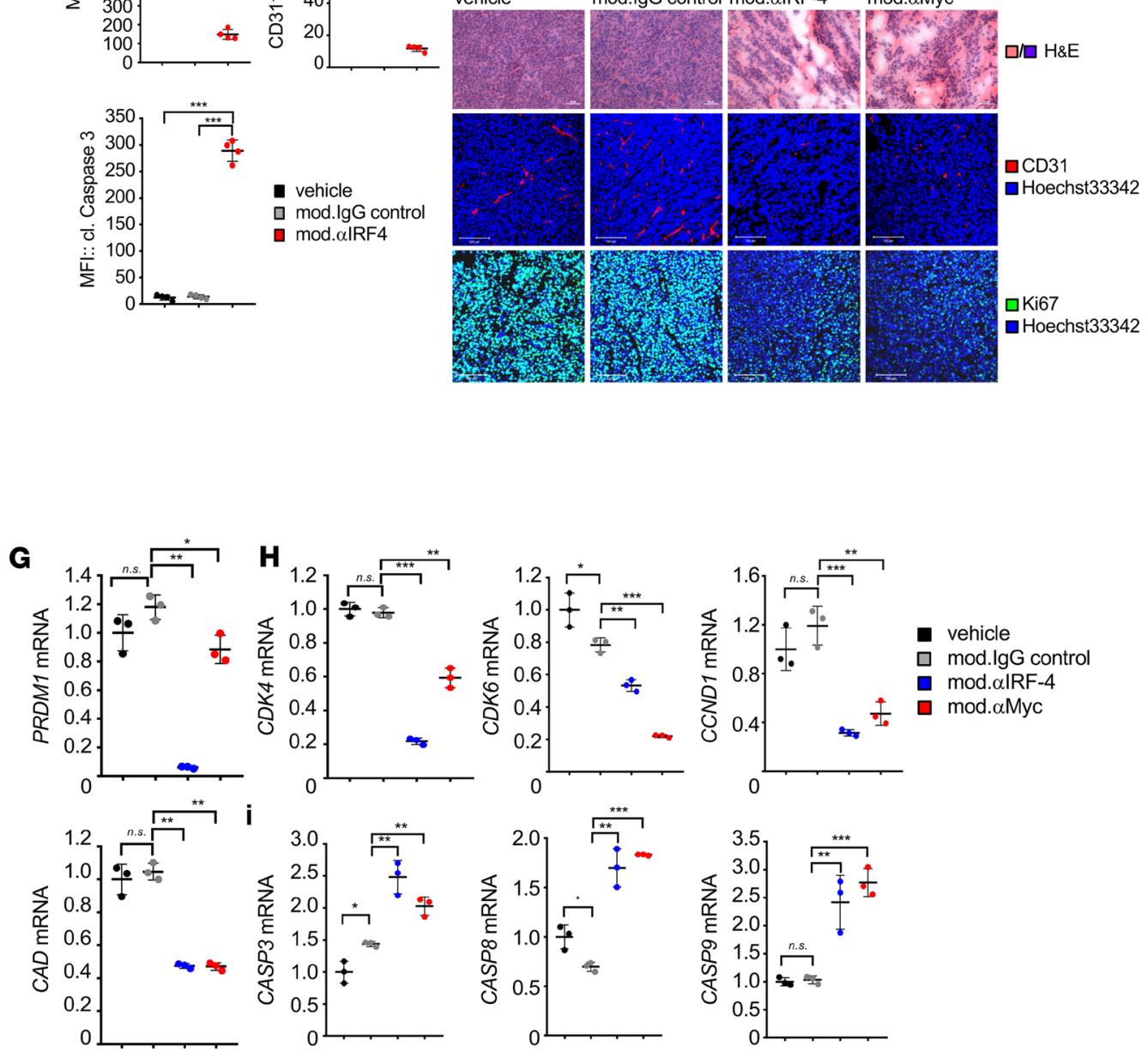
Figure 4. Antitumor effects by local or systemic administration of PS DNA oligo-modified IRF4 or Myc antibodies in vivo. (A) Effects on tumor growth kinetics and tumor tissues by PS DNA oligo-modified antibody local treatments ( $n=7$ ). Scale bars: $50 \mu \mathrm{m}, 100 \mu \mathrm{m}$. (B) IRF4 nuclear accumulation using nuclear extracts prepared from indicated tumors. (C) Growth kinetics of MM.1S tumors treated systemically by the indicated modified antibodies ( $n=$ 4). (D) Oligo pull-down assay showing loss of DNA-binding activity of IRF4 and Myc transcription factors targeted by modified antibodies in vivo. (E) Effects on tumor tissues of systemic treatments of the indicated modified antibodies. Scale bar: $100 \mu \mathrm{m}$. (F) Quantification of images shown in E and G, with RT-PCR measuring expression of IRF4 and Myc target genes in tumors grown in mice systemically treated by the indicated modified antibodies. (H) Expression levels of genes critical for cell cycle regulation and (I) tumor cell apoptosis, upon systemic treatments with indicated modified antibodies, performed in triplicate. (A and C) SD shown, 2-way ANOVA (Tukey's multiple-comparisons test): ${ }^{*} P<0.01$, and ${ }^{* * *} P<0.001$. (B, F, and $\mathbf{C}$ ) SD shown. Unpaired Student's $t$ test: n.s., not significant, ${ }^{*} P<0.05,{ }^{* *} P<0.01$, and ${ }^{* * *} P<0.001$.

tumors. Although there is much to be further explored, our data so far also indicate that blocking efflux, and/or lysosomal degradation, enables partial intracellular retention of the modified nontargeting antibodies. These properties of the cell-penetrating antibodies - requirement of antigen/target for cellular retention and fluxing both directions - are unique. They enable the use of the cell-penetrating antibodies as research tools, diagnostics, and more efficient therapeutics.

Much remains to be learned about the permeability barrier properties of cell membranes before rationally designing approaches to open such barriers to allow the passage of macromolecules through biomembranes, especially without being trapped in endosomes. However, fundamental discoveries in nuclear membrane properties that allow import and export of macromolecules in the size range comparable to or larger than antibodies may shed light on how our cell-penetrating antibodies overcome the permeability barrier of cell and nuclear membranes. It has been shown that nuclear core complexes, although disallowing passage of cargoes greater than $30 \mathrm{kDa}$, permit rapid passage of inert macromolecules bound to karyopherin transport receptors. It has been suggested and supported by experimental evidence that the permeability barrier of the nucleus has a sieve structure and that nuclear pore proteins form a 3D meshwork with hydrogel-like properties (41). Importantly, nuclear transport receptors can, by disrupting adjacent interrepeat contact of the meshwork, transiently open adjoining meshes, thereby enabling the passage of large molecules (41). It is conceivable that a cell biomembrane also has the reversible sieve structure and it can be transiently opened if the mesh sieve is disrupted by increased membrane potential. Although more studies are required to determine the detailed mechanism(s) by which PS DNA oligonucleotides facilitate antibody cell membrane penetration, our results suggest that the cell penetration of the modified antibodies requires an increase in the membrane potential. The anionic nature of PS DNA oligos may also play a role in changing the membrane potential, conceivably also by facilitating interaction with the cell membrane, leading to the modified antibodies' cell penetration. Anionic nanoparticles are known to associate with membranes and could enhance cell penetration $(42,43)$. Nevertheless, further experiments are needed to prove and to fully understand the mechanisms underlying PS DNA oligo-modified antibody cell penetration.

Binding to a target protein facilitates intracellular retention of the modified antibodies, in part, preventing their cellular egress. The binding of modified antibodies to the target protein is also likely critical in reducing lysosomal degradation of the antibodies, conceivably partially by keeping them in the locations where the antigens reside.

The cell-penetrating antibody technology we describe here allowed us to quickly generate an antibody-based inhibitor for transcription factors, i.e., IRF4 and Myc. We also showed the ability of an anti-tyrosine antibody for Src to inactivate Src tyrosine kinase by masking the active site of the kinase. However, the antitumor potency of any treatment modality depends on the effectiveness of the drug candidate in inhibiting the target, as well as the importance of the target for the survival and proliferation of the cancer cells in vivo. Although treating tumor-bearing mice with the modified IRF4, Myc, and pY(418)-Src antibodies led to tumor growth inhibition, these antibodies were not designed or optimized to be inhibitors of their targets - they are commercially available antibodies characterized for ELISA-based recognition of native proteins. For clinical translation, much remains to be tested to generate effective antibody-based drugs. They include development of antibodies of totally human sequences, then screening and selecting the most potent antagonistic or agonistic antibody for the target to have beneficial effects. In addition, optimal dosing needs to be determined. In our in vivo studies described here, the highest dosage used was $10 \mu \mathrm{g}$ per treatment, which is at least 10 -fold lower than many other antibody therapies routinely used for inhibiting cell surface receptors to block tumorigenesis in mouse tumor models $(9,44,45)$, including the recently reported cell-penetrating RAS antibody (19). The cell-penetrating RAS antibody has also illustrated that further modification of a cell-internalizing antibody with a moiety, such as tumor-associated integrin, for tumor tissue targeting can markedly improve antitumor effects (19). 


\section{A}
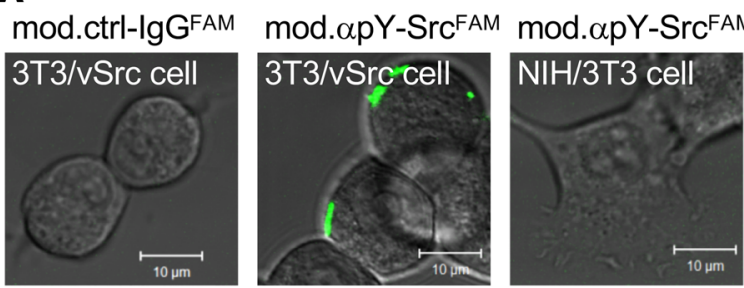

$\square$ mod.antibody 口DIC

\section{B}

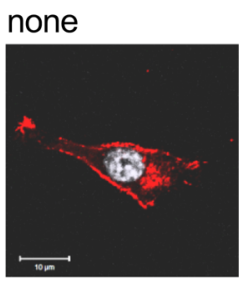

unmod. $\alpha p Y-S r c$
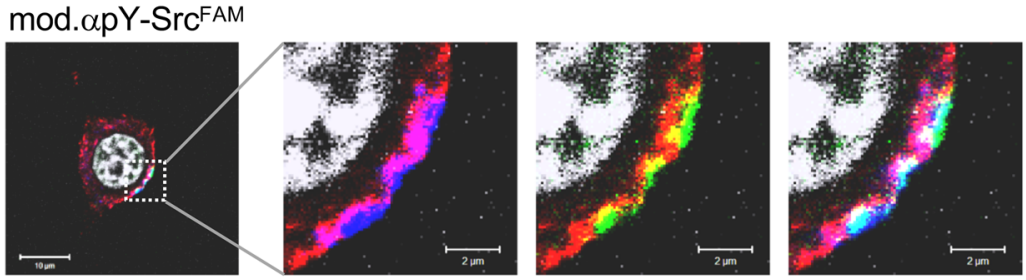

$\square$ pY-Src protein

$\square$ FAM-oligossDNA $]$ mod. apY-Src

$\square$ Hoechst33342
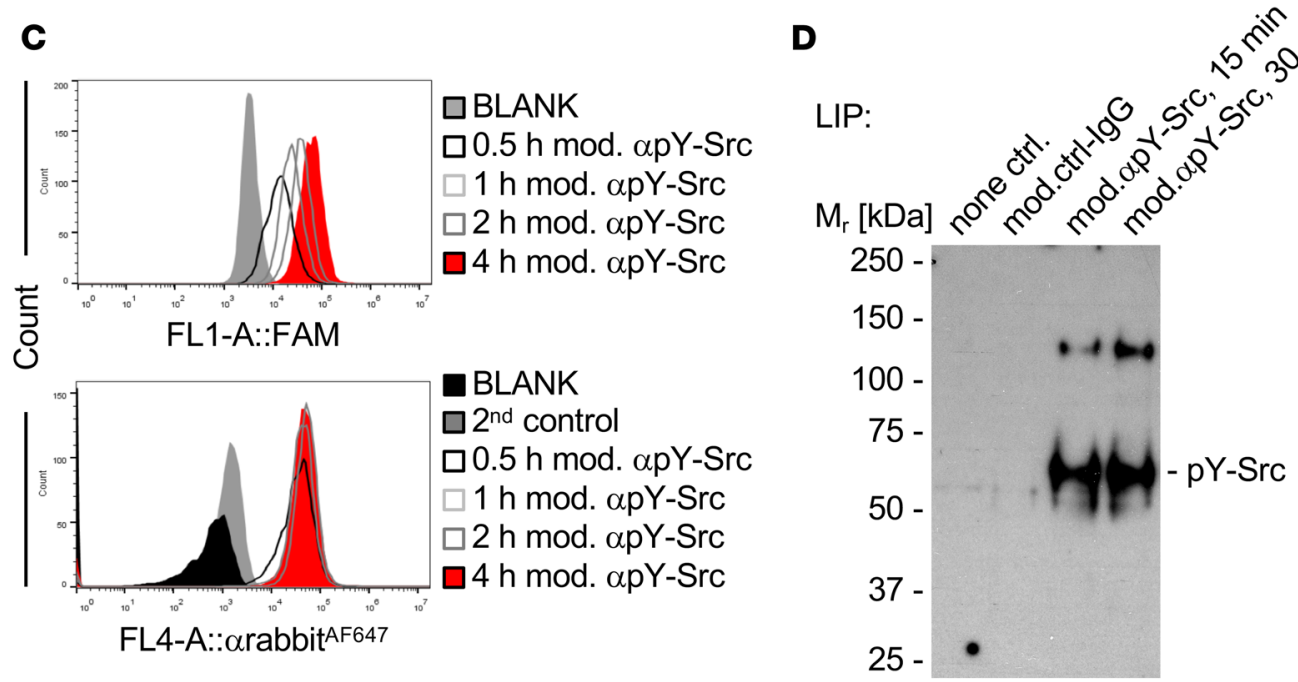

$E$

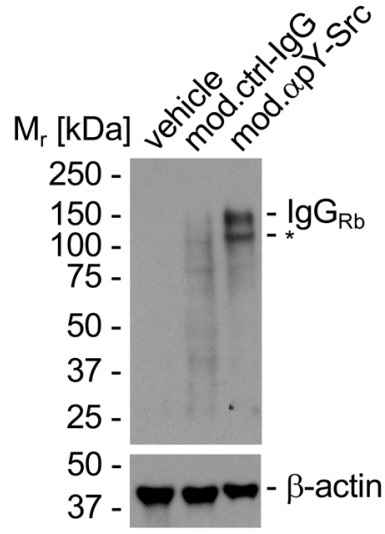

Figure 5. An antibody Src kinase inhibitor. (A) Representative live cell imaging showing spatially confined localization of PS DNA oligo-modified pY(418)-Src antibodies at the inner cell membrane in indicated cells. DIC, differential interference contrast. (B) Confocal microscopy showing the PS DNA oligo-modified anti-pY(418)-Src antibody colocalized with its antigen, pY(418)-Src kinase. Scale bars: $10 \mu \mathrm{m}, 2 \mu \mathrm{m}$. (C) Representative flow cytometry detecting fluorescent label (upper) or IgG (lower) to show cellular internalization kinetics of PS DNA oligo-modified pY(418)-Src antibody. (D) Representative LIP/Western blot demonstrating the modified $\mathrm{pY}(418)$-Src antibody antigen recognition at indicated time points after antibody incubation. (E) In vivo biostability of PS DNA oligo-modified antipY(418)-Src antibody (24 hours after last treatment) shown by Western blot using tissue homogenates isolated from tumors treated as indicated.

In addition to generating antibody-based therapeutics for intracellular targets, we also demonstrated the feasibility of this platform to detect living, cytokine-producing $\mathrm{CD} 8^{+} \mathrm{T}$ cells, overcoming a major hurdle for immune monitoring and isolating living immune cells that are either immune activating or suppressing for adoptive transferring of $\mathrm{T}$ cells or other clinical uses. Our extensive data also demonstrated the capacity of the technology to create research tools, allowing detection and analyses of intracellular molecules in their native form with their natural interacting partners in living cells. Taken together, we have developed a technology platform to effectively deliver antibodies and likely other proteins, including peptides and enzymes, into cells for drug development, diagnostics, and research. 


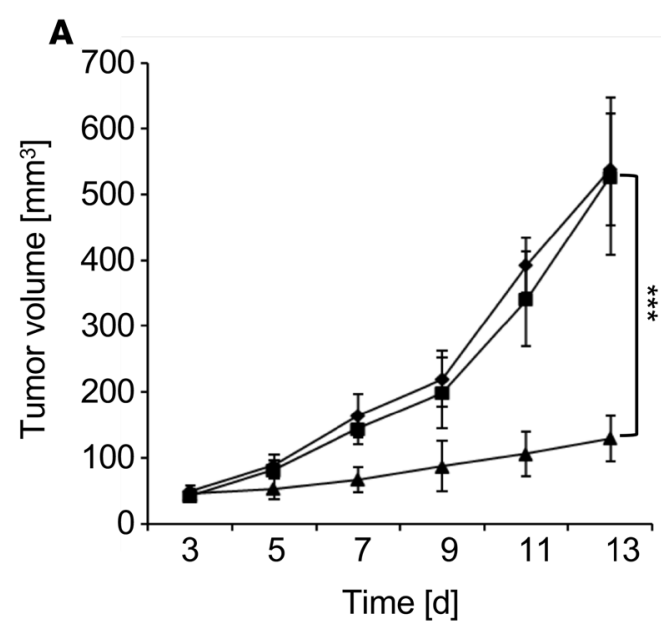

vehicle, $n=8$

- mod.lgG control, $n=8$

$\Delta$ mod.apY418Src, $n=8$
D

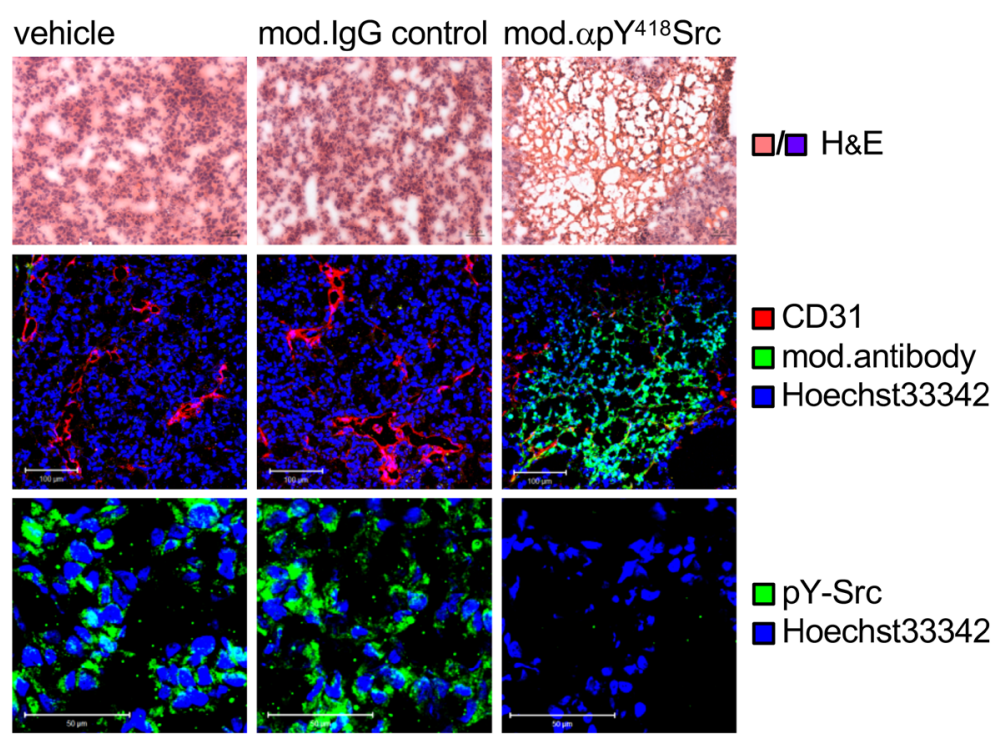

B

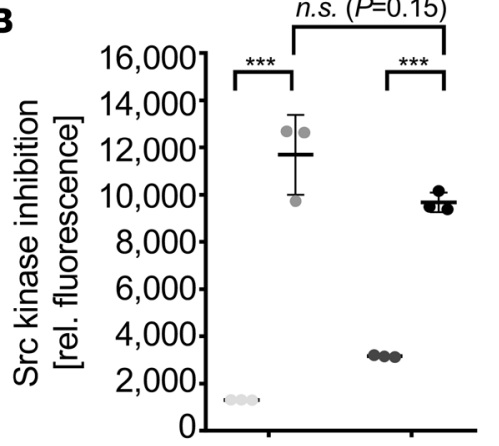

$\square$ unmod.lgG control

$\square$ unmod.apY418Src

$\square$ mod.lgG control

- mod.apY418Src
C

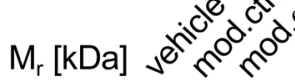

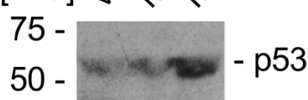

50 -

37 -

$20-$

15 -

$50-$

$37-$

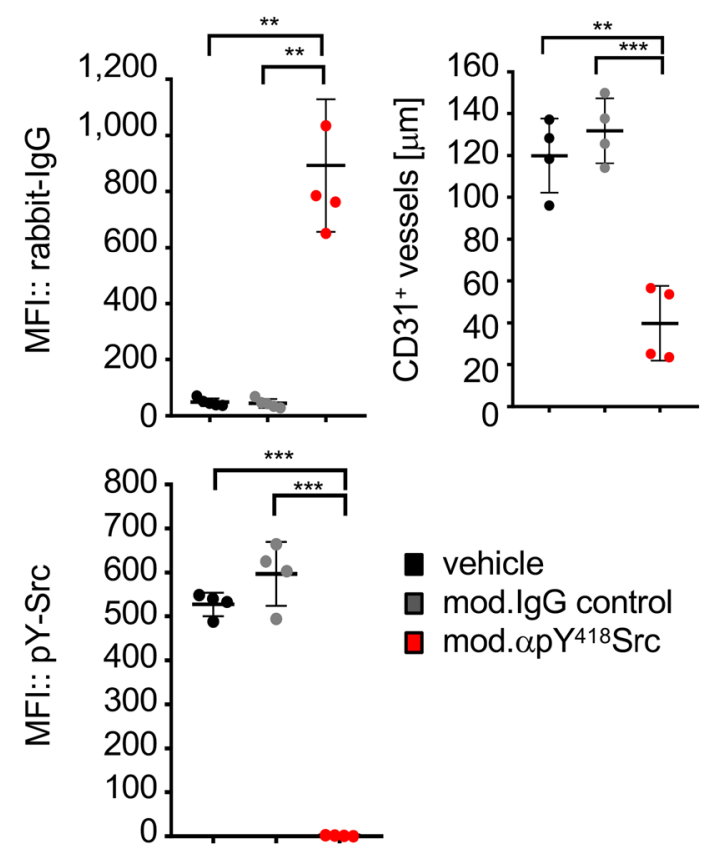

E
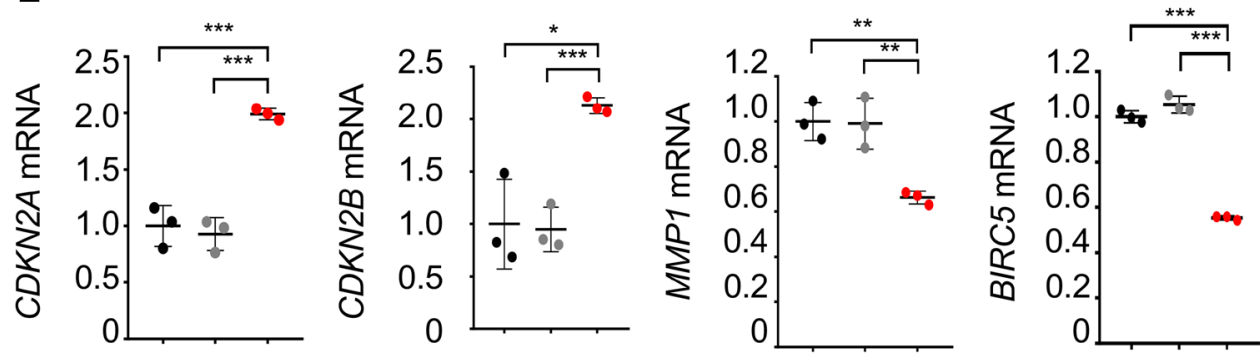

vehicle

$\square$ mod.lgG control

$\square$ mod.apY418Src 
Figure 6. Antitumor effects by PS DNA oligo-modified pY(418)-Src antibody. (A) Tumor growth kinetics when treated with indicated modified antibodies $(n=8)$. SD shown. Two-way ANOVA (Tukey's multiple-comparisons test) was used: ${ }^{* *} P<0.001$. (B) ELISA on pY(418)-Src recognition by anti-pY(418)Src antibody upon modification performed twice in triplicates. (C) Representative Western blot assessing gene expression in tissue homogenates isolated from tumors. (D) Tumor tissue integrity, tumor accumulation of modified antibodies and CD31+ tumor vasculature, and pY(418)-Src levels shown by confocal microscopy (left) with quantification (right). (E) Representative RT-PCR on Src kinase target gene mRNA expression in tumors treated as indicated, performed in triplicates. (B, D, and E) SD shown. Unpaired Student's $t$ test: ${ }^{*} P<0.01$, and ${ }^{* *} P<0.001$.

\section{Methods}

\section{Mice and cell culture}

For subcutaneous tumor challenge, athymic $n u / n u$ mice (NCI Frederick) were engrafted with up to $5 \times$ $10^{6} \mathrm{MM}$.1S human multiple myeloma or $5 \times 10^{6}$ human melanoma A2058 cells s.c. into the flank. Once tumors reached 5 to $7 \mathrm{~mm}$ in diameter, local and systemic treatments with $10 \mu \mathrm{g} / \mathrm{dose} / \mathrm{mouse}$ modified antibody were administered every other day (for MM.1S) or every day (for A2058).

MM.1S cells (gift of Steve J. Forman, Department of Hematology and Hematopoietic Cell Transplantation, Comprehensive Cancer Center at City of Hope National Medical Center, Duarte, California, USA) were cultured in RPMI1640 (Gibco) supplemented with 10\% FBS (MilliporeSigma), 1\% GlutaMax (Gibco), 25 mM HEPES, human myeloma H929 (ATCC) supplemented with 10\% FBS, and 0.05 mM mercaptoethanol. Human lymphoma Ly10 (gift of Richard Jove, Department of Molecular Medicine, Beckman Research Institute at the Comprehensive Cancer Center at City of Hope National Medical Center, Duarte, California, USA) were cultured in IMDM (Gibco) supplemented with 20\% FBS. A2058 (ATCC), genetically engineered MEF cell lines, murine fibroblasts NIH 3T3 and 3T3/vSrc (gift of Richard Jove), and human colon carcinoma HCT116 (ATCC) were grown in DMEM (Gibco) containing 10\% FBS. Human breast adenocarcinoma cell lines MCF7, MDA-MB-231, MDA-MB-468, and MDA-MB-435 (ATCC) were cultured in DMEM (Gibco) supplemented with 10\% FBS (MilliporeSigma). Primary murine lymphocytes isolated from spleen were cultured in IMDM supplemented with 10\% FBS and $0.05 \mathrm{mM}$ mercaptoethanol.

\section{Conjugation chemistry for modified antibodies}

Conjugation chemistry was carried out by LACell Therapeutics Inc. within Sorrento Therapeutics. Briefly, a fully PS ssDNA 20-mer was extended on its 5 ' end by $4 \times$ C3 (propyl) spacers/linker ending in a DBCOTEG reactive group to facilitate conjugation to antibodies via click chemistry. PS ssDNA oligos entail-

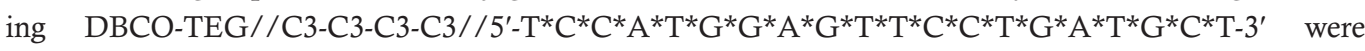
obtained from TriLink (asterisks mark phosporothioation). Purification of modified antibodies revealed a conjugate of 1:1 or 1:2 antibody/oligo ratio (Supplemental Figure 2).

The following antibodies used were purchased from Santa Cruz Biotechnology: anti-tubulin, mouse IgG sc-398103, anti-IRF4, mouse IgG sc-48338, anti-Myc (clone 9E10), mouse IgG sc-40, anti-pY419. Src, rabbit IgG sc-139601, normal mouse IgG sc-2025, and normal rabbit IgG sc-2027. Anti-IFN- $\gamma$ (clone AN-18) and normal rat IgG control (clone RTK2071) were purchased from BioLegend.

\section{Modified antibody purification}

Protein A purification. Excess oligos were removed through affinity chromatography using protein A resin (MabSelectSure, GE Healthcare). Crude oligo-antibody conjugates were eluted with 10 column volumes (CVs) of $100 \mathrm{mM}$ glycine, $\mathrm{pH} 2.8$, and immediately neutralized with 10\% $1 \mathrm{M}$ Tris-HCl, $\mathrm{pH}$ 8.0. Purified oligo-antibody conjugates were buffer exchanged with $1 \times$ Dulbecco's PBS (DPBS) and subsequently concentrated using Amicon centrifugal filter (MilliporeSigma, $30 \mathrm{kDa} \mathrm{MWCO}$ ) at $1500 \mathrm{~g}, 4^{\circ} \mathrm{C}$.

Hydrophobic-interaction chromatography purification via ÄKTA fast protein liquid chromatography. Protein Apurified oligo-antibody conjugates were purified, isolating +1 oligo- and +2 oligo-modified antibodies. Samples were (a) diluted 2-fold with 1.5 M ammonium sulfate/50 mM sodium phosphate, $\mathrm{pH} 7.0$, adjusting the initial salt concentration to $0.7 \mathrm{M}$ ammonium sulfate and allowing binding to the column, and (b) loaded onto a Butyl Sepharose High Performance (GE Healthcare) column and eluted with a $0 \%-100 \%$ linear gradient of hydrophobic interaction chromatography (HIC) ÄKTA Buffer B (50 mM sodium phosphate/10\% isopropyl alcohol, pH 7.0; HIC ÄKTA Buffer A: 750 mM ammonium sulfate/50 mM sodium phosphate, pH 7.0) for $35 \mathrm{CVs}$ using ÄKTA fast protein liquid chromatography (FPLC, GE Healthcare). Fractions corresponding to +1 oligo- and +2 oligo-modified antibodies were combined, buffer exchanged with $1 \times$ DPBS, and con- 
centrated using Amicon centrifugal filter (MilliporeSigma, $30 \mathrm{kDa} \mathrm{MWCO}$ ) at $1500 \mathrm{~g}, 4^{\circ} \mathrm{C}$. Conjugates were injected into HIC and size-exclusion chromatography (SEC) HPLC to confirm identities.

Ion exchange purification via ÄKTA FPLC. Protein A-purified oligo-antibody conjugates were diluted 10-fold with ion exchange (IEX) Buffer A (50 mM Tris/100 mM NaCl, pH 8) and loaded onto a Capto Q ImpRes column (GE Healthcare). Sample elution with a $0 \%-100 \%$ linear gradient of IEX ÄKTA Buffer B (50 mM Tris/1.5 M NaCl, pH 8) for 35 CVs using ÄKTA FPLC (GE Healthcare). Fractions corresponding to +1 oligo- and +2 oligo-modified antibodies were combined, buffer exchanged with $1 \times$ DPBS, and concentrated using Amicon centrifugal filter (MilliporeSigma, $30 \mathrm{kDa} \mathrm{MWCO}$ ) at $1500 \mathrm{~g}$, $4^{\circ} \mathrm{C}$. Conjugates were injected into HIC and SEC HPLC to confirm identities.

\section{Characterization of purified modified antibody}

HIC HPLC analysis. DNA oligo-antibody conjugates were analyzed via HIC using 1260 Affinity HPLC (Agilent Technologies) and TSKgel Butyl-NPR analytical column $(4.6 \mathrm{~mm}$ ID, $2.5 \mu \mathrm{m} \times 3.5 \mathrm{~cm}$, Tosoh Bioscience) at a linear gradient of $5 \%-85 \%$ Buffer B for 30 minutes with $1.0 \mathrm{M}$ ammonium sulfate $/ 50 \mathrm{mM}$ sodium phosphate, $\mathrm{pH} 7.0$, as HIC HPLC Buffer A and $50 \mathrm{mM}$ sodium phosphate $/ 25 \%$ isopropanol, $\mathrm{pH}$ 7.0 (HIC HPLC Buffer B).

IEX HPLC analysis. Oligo-antibody conjugates were analyzed using 1260 Affinity HPLC (Agilent Technologies) and Proteomix SAX-NP3 $(4.6 \mathrm{~mm} \times 50 \mathrm{~mm}, 3 \mu \mathrm{m}$, Sepax) at a linear gradient of $0 \%-100 \%$ Buffer B for 20 minutes with 50 mM Tris, pH 8 (IEX HPLC Buffer A), and $50 \mathrm{mM}$ Tris/1.5 M NaCl, pH 8 (IEX HPLC Buffer B).

SEC HPLC analysis. Conjugates were analyzed using 1260 Affinity HPLC (Agilent Technologies) and TSKgel G3000SWXL column $(7.8 \mathrm{~mm}$ ID, $5 \mu \mathrm{m} \times 30 \mathrm{~cm})$ run for 30 minutes with $1 \times$ DPBS.

$S D S-P A G E$ analysis. For SDS-PAGE analysis, $2.5 \mu \mathrm{g}$ of oligo-antibody conjugates suspended in loading buffer (NuPAGE, Thermo Fisher Scientific) were separated on a 4\%-12\% Bis-Tris SDS-PAGE (Thermo Fisher Scientific) under nonreducing and reducing conditions (treated with $1 \times$ reducing agent by NuPage, Thermo Fisher Scientific, and heated for 10 minutes at $70^{\circ} \mathrm{C}$ ) compared with unmodified antibodies. For protein separation, $1 \times$ MOPS buffer (Life Technologies) was used as running buffer at $\mathrm{V}=180$ volts for 50 minutes. A protein ladder (Mark 12, Thermo Fisher Scientific) was loaded to determine molecular weight of antibodies. SDS-PAGE was stained using SYPRO Ruby (Invitrogen) overnight according to the manufacturer's instructions. Images were acquired on a ChemiDoc (Bio-Rad) by detecting SYPRO dye and Alexa Fluor 488 emission.

Bicinchoninic acid analysis

Concentration of conjugates was determined by bicinchoninic acid analysis (Thermo Fisher Scientific) using bovine gamma globulin as the standard according to the manufacturer's instructions.

\section{LIP assay}

For testing (a) cell permeation activity and (b) recognition of intracellular antigens by modified antibodies, cells were incubated with up to $10 \mu \mathrm{g} / \mathrm{mL}$ modified antibodies for up to 4 hours with living cells. Cells were washed thoroughly 3 times with PBS before lysis. Once cells went through whole-cell lysis using RIPA lysis buffer containing $50 \mathrm{mM}$ Tris (pH 7.4), $150 \mathrm{mM} \mathrm{NaCl}, 1 \mathrm{mM}$ EDTA, 0.5\% NP-40, $1 \mathrm{mM} \mathrm{NaF}, 15 \%$ glycerol, and $20 \mathrm{mM} \beta$-glycerophosphate (protease inhibitor cocktail was added fresh to lysis buffer [Mini Protease Inhibitor Cocktail, Roche]), lysates were cleared from debris, and $30 \mu \mathrm{L}$ protein $\mathrm{G}$-coated agarose beads (Invitrogen) were added to up to $500 \mu \mathrm{L}$ cell lysate to engage modified antibodies. Immunoprecipitation was carried out at $4^{\circ} \mathrm{C}$ shaking overnight, before precipitates were washed thrice with ice-cold PBS, $30 \mu \mathrm{L}$ Laemmli buffer was added, and precipitates were boiled for 5 minutes at $95^{\circ} \mathrm{C}$ and subsequently subjected to electrophoretic protein separation by SDS-PAGE, followed by Western blotting and immunodetection using antitubulin (rat IgG, sc-53030), anti-IRF4 (goat IgG, sc-6059), or anti-pY(418)-Src (mouse IgG, sc-81521).

\section{Cellular internalization and cellular clearance studies}

To assess cellular internalization efficacy, membrane potential of cells was depolarized using $120 \mathrm{mM} \mathrm{KCl}$ (final) preincubation before cells were treated for 1 hour with $5 \mu \mathrm{g} / \mathrm{mL}$ modified antibodies.

The human Fc $\gamma$ R-binding inhibitors were obtained from Thermo Fisher Scientific (catalog 16-9161-73 and 14-9161-71) and used as recommended by the manufacturer's instructions. Inhibitors blocking cellular endocytic activity were used at $50 \mu \mathrm{g} / \mathrm{mL}$ dextran (scavenger receptors, MilliporeSigma), $100 \mu \mathrm{M}$ cadav- 
erine (clathrin-mediated endocytosis, MilliporeSigma), $2 \mu \mathrm{g} / \mathrm{mL}$ filipin (caveolae-mediated endocytosis, MilliporeSigma), $150 \mu \mathrm{M}$ genistein (lipid raft-mediated endocytosis, MilliporeSigma), $1 \mathrm{mM}$ amiloride (micropinocytosis, MilliporeSigma), and $50 \mu \mathrm{g} / \mathrm{mL}$ chondroitin (control, MilliporeSigma).

Cellular clearance by efflux and lysosomal degradation was assayed using $100 \mathrm{mg} / \mathrm{mL}$ verapamil hydrochloride (MilliporeSigma) and $50 \mu \mathrm{M}$ chloroquine (MilliporeSigma) preincubated for 2 hours before cells were treated with $5 \mu \mathrm{g} / \mathrm{mL}$ modified antibodies. Cellular clearance by efflux was allowed for $45 \mathrm{~min}$ utes, with the supernatant exchanged every 15 minutes, followed by resuspending the cells in staining buffer (PBS $+1 \%$ BSA, MilliporeSigma) for flow cytometric analysis.

\section{Phenotypic detection of cytokine-producing lymphocytes}

Lymphocytes were isolated from spleens of immune-competent C57BL/6 mice, and erythrocytes were lysed (Red Cell Lysis Buffer, MilliporeSigma) before splenocytes were cultured for 6 hours with the modified anti-IFN- $\gamma$ or control IgG antibodies. IFN- $\gamma$ production by splenic cell populations was stimulated using $5 \mathrm{ng} / \mathrm{mL}$ PMA and $500 \mathrm{ng} / \mathrm{mL}$ ionomycin (MilliporeSigma) including monensin solution (BioLegend) and incubated with $5 \mu \mathrm{g} / \mathrm{mL}$ modified antibodies for 6 hours at $37^{\circ} \mathrm{C}$. For additional immunostainings, cells were harvested at $524 \mathrm{~g}$ centrifugation for 5 minutes and washed twice with PBS. To exclude dead cells from analysis, cells were stained with LIVE/DEAD Fixable Aqua Dead Cell Stain (Invitrogen) according to the manufacturer's instructions, followed by washing with PBS before staining $\mathrm{CD} 8^{+} \mathrm{T}$ cells using anti-CD8 antibody (BioLegend) suspended in Fc- $\gamma$ block (diluted $1: 100$ ) containing staining buffer (PBS $+1 \%$ BSA, MilliporeSigma). For intracellular staining, cells were resuspended after 1 PBS wash in $200 \mu \mathrm{L}$ Fix/Perm solution (BioLegend) for 20 minutes at room temperature and washed with Perm buffer according to the manufacturer's instructions (BioLegend). Intracellular IFN- $\gamma$ was additionally stained using anti-IFN- $\gamma$-PE (clone XMG1.2, BioLegend), resuspended in staining buffer, and incubated for 30 minutes on ice before cells were washed and resuspended for analysis by flow cytometry.

\section{Imaging}

Confocal imaging of fixed as well as living cells was carried out using an LSM 510 Meta microscope (Zeiss). Samples were prepared by treating living cells with either $10 \mu \mathrm{g} / \mathrm{mL}$ (modified anti-IRF4 and pY[418]-Src) or $100 \mu \mathrm{g} / \mathrm{mL}$ (modified antitubulin) FAM-labeled, modified antibodies for up to 2 hours. Subsequently, cells were fixed and prepared for indirect immunofluorescence imaging as described previously (46) using antitubulin (rat IgG) and anti-IRF4 (goat IgG) for intracellular staining. The tubulin cytoskeletal fiber network was analyzed by collecting 3D Z-stacks shown collapsed to 2D images ("through-focus") using software provided by Zeiss. Indication of tubulin/modified antitubulin colocalization was assessed using the crosshair function on scatter grams of pixel-by-pixel distribution. Images double positive for used fluorophores were generated by selecting the upper-right quadrant of the scatter gram using software provided by Zeiss. For live cell imaging, cells were grown on glass-bottom dishes and treated with $10 \mu \mathrm{g} / \mathrm{mL}$ modified antibody for 1.5 hours before live cell imaging was carried out in a thermostated chamber at $37^{\circ} \mathrm{C}, 5 \% \mathrm{CO}_{2}$.

For immunostainings, antibodies raised against Ki67 (Cell Signaling Technology), CD31 (BD Pharmingen), cleaved caspase-3 (Cell Signaling Technology), mouse IgG, rabbit IgG (both from Amersham), and pY(418)-Src (Santa Cruz Biotechnology) were used.

To assess whether the modified antibodies were on targets in tumors after systemic treatments by microscopy, OCT-frozen tumor tissues were fixed in 2\% formaldehyde and permeablized with methanol at $-20^{\circ} \mathrm{C}$ for 15 minutes and blocked in PBS containing $10 \%$ albumin for 1 hour at room temperature. Modified Myc antibody-treated tumors were stained with rabbit anti-Myc (D84C12, Cell Signaling Technology 5605) and goat anti-FITC (also reacts with FAM, Abcam ab19224). Modified IRF4 antibody-treated tumors were stained with goat anti-IRF4 (Santa Cruz Biotechnology sc-6059) and rabbit anti-FITC (also reacts with FAM, Thermo Fisher Scientific 71-1900). All samples were stained at $4^{\circ} \mathrm{C}$ overnight, and Alexa Fluor-labeled secondary antibodies (Thermo Fisher Scientific) were used to stain samples after 3 washes with PBS. The nuclei were visualized with Hoechst 33342 staining (MilliporeSigma). Slides were then mounted and analyzed by confocal microscopy. The confocal imaging was carried out using a $\times 20$ immersion objective on an LSM 700 confocal microscope (Zeiss). 


\section{Immunoblotting}

Whole-cell lysates as well as tumor tissue homogenates were prepared using RIPA lysis buffer containing $50 \mathrm{mM}$ Tris (pH 7.4), $150 \mathrm{mM} \mathrm{NaCl}, 1 \mathrm{mM}$ EDTA, 0.5\% NP-40, $1 \mathrm{mM} \mathrm{NaF}, 15 \%$ glycerol, and 20 $\mathrm{mM} \beta$-glycerophosphate. A protease inhibitor cocktail was added fresh to the lysis buffer (Mini Protease Inhibitor Cocktail, Roche). Normalized protein amounts were subjected to electrophoretic separation by SDS-PAGE, transferred onto nitrocellulose for Western blotting, and subsequently immunodetected using antibodies against tubulin, IRF4, Myc, pY(418)-Src, cleaved PARP, p53, cleaved caspase-3, Src (all from Cell Signaling Technology), HDAC1, and $\beta$-actin (both from MilliporeSigma).

\section{Electrophoretic mobility shift assay}

Target DNA binding of protein was assessed by electrophoretic mobility shift assay. Briefly, nuclear extracts isolated from human multiple myeloma cells (MM.1S) were incubated with a Myc-responsive E-box containing dsDNA oligos encoded by ${ }^{32} \mathrm{P}$-radiolabeled E-box forward (F), 5'-AGTTGACCACGTGGTCTGGCCACGTGGTCTGGG-3', and E-box reverse (R), 5'-CCCAGACCACGTGGCCAGACCACGTGGTCAACT-3'. Nuclear extracts were isolated using buffer A (10 mM HEPES/KOH at $\mathrm{pH} 7.9,1.5 \mathrm{mM}$ $\left.\mathrm{MgCl}_{2}, 10 \mathrm{mM} \mathrm{KCl}\right)$ and buffer C (20 mM HEPES/KOH at pH 7.9, $420 \mathrm{mM} \mathrm{NaCl}, 1.5 \mathrm{mM} \mathrm{MgCl}, 0.2$ $\mathrm{mM}$ EDTA, 25\% glycerol); per $2 \mathrm{~mL}$ buffer A or C, protease inhibitors at $0.2 \mathrm{mM} \mathrm{PMSF}, 0.5 \mathrm{mM} \mathrm{DTT}$, and $1 \mathrm{mM} \mathrm{Na} \mathrm{VO}_{4}$ were added fresh before use. Cells were washed with PBS, resuspended in buffer $\mathrm{A}$, incubated on ice for 20 minutes, and sedimented by centrifugation for 20 seconds at $16,100 \mathrm{~g}$ in a tabletop centrifuge. Pellet was resuspended in buffer $\mathrm{C}$, incubated for 30 minutes on ice, and sedimented by centrifugation for 10 minutes at 16,100 g. dsDNA Myc/E-box oligo containing a Myc consensus binding site was radiolabeled by $5^{\prime}$ end labeling using $\left[\gamma_{-}{ }^{32} \mathrm{P}\right]$ ATP (PerkinElmer) and T4 polynucleotide kinase (New England BioLabs). Nuclear extracts were resuspended at $10 \mu \mathrm{g}$ with loading buffer (50 mM HEPES at $\mathrm{pH}$ $7.8,5 \mathrm{mM}$ EDTA at $\mathrm{pH} 8,25 \mathrm{mM} \mathrm{MgCl}_{2}$ adjusted to $\mathrm{pH} 7.8$ with $3 \mathrm{M} \mathrm{KOH}$ ) containing radiolabeled Myc/ E-box oligo and separated by PAGE; dried gel was exposed on x-ray film to assess Myc DNA binding. For supershift analysis, $\alpha$-Myc antibody (Cell Signaling Technology D84C12) was added to nuclear extract at $1 \mu \mathrm{g} / 20 \mu \mathrm{L}$ and incubated on ice for 15 minutes before loading onto PAGE for electrophoretic separation.

\section{DNA oligo pull-down assay}

To assess the DNA-binding capacity of the transcription factors IRF4 and Myc, oligo pull-down assays were performed using biotinylated dsDNA oligos (IDT) harboring IRF4 or Myc consensus binding sites: IRF4 F, 5'-TAAGAGGAAGTGAAAGTCCC-3'-biotin, IRF4 R, 5'-GGGACTTTCACTTCCTCTTA-3'-biotin; Myc F, 5'-AGTTGACCACGTGGTCTGGCCACGTGGTCTGGG-3'-biotin, Myc R, 5'-CCCAGACCACGTGGCCAGACCACGTGGTCAACT-3'-biotin. Briefly, nuclear protein extracts were isolated from tumors using extraction buffer A containing $10 \mathrm{mM} \mathrm{HEPES-KOH}$ at $\mathrm{pH} 7.9,1.5 \mathrm{mM} \mathrm{MgCl}_{2}$, and $10 \mathrm{mM}$ $\mathrm{KCl}$, after incubating for 10 minutes on ice. Tissue/cell pellet sedimented at $16,100 \mathrm{~g}$ for 20 seconds at $4^{\circ} \mathrm{C}$; was resuspended in high-salt extract buffer B containing $20 \mathrm{mM}$ HEPES- $\mathrm{KOH}$ at pH 7.9, $1.5 \mathrm{mM} \mathrm{MgCl}_{2}$, $420 \mathrm{mM} \mathrm{NaCl}, 0.2 \mathrm{mM}$ EDTA, and 25\% glycerol; and was incubated for 20 minutes on ice. Supernatants were collected upon sedimentation at $16,100 \mathrm{~g}$ for 2 minutes at $4^{\circ} \mathrm{C}$. Protease inhibitors were added fresh to extraction buffers A and C ( $0.2 \mathrm{mM}$ PMSF, $0.5 \mathrm{mM}$ DTT, $\left.1 \mathrm{mM} \mathrm{Na}_{3} \mathrm{VO}_{4}\right)$. Then, $250 \mu \mathrm{g}$ protein/sample of nuclear extract was incubated for 2 hours at room temperature in DNA oligo-binding buffer (12 mM HEPES- $\mathrm{KOH}$ at $\mathrm{pH} 7.9,12 \%$ glycerol, $4 \mathrm{mM}$ Tris- $\mathrm{HCl}$ at $\mathrm{pH} 7.9,150 \mathrm{mM} \mathrm{KCl}, 1 \mathrm{mM}$ EDTA; added fresh: $1 \mathrm{mM} \mathrm{DTT}, 0.1 \mathrm{mg} / \mathrm{mL}$ poly[deoxyinosinic-deoxycytidylic], $0.5 \mathrm{mg} / \mathrm{mL} \mathrm{BSA}$ ) and $2 \mu \mathrm{g}$ biotinylated dsDNA oligo at room temperature, gently shaking. Paramagnetic particles ( $50 \mu \mathrm{L} /$ sample Streptavidin MagneSphere, Promega) were washed twice with DNA oligo-binding buffer and blocked using $100 \mu \mathrm{g}$ BSA, 10 $\mu \mathrm{g}$ poly(deoxyinosinic-deoxycytidylic), and $10 \mu \mathrm{g}$ salmon sperm DNA for 30 minutes at room temperature, gently shaking. Prewashed and blocked magnetic particles were added to the nuclear extract/DNA oligo mix and incubated for 2 hours at room temperature, gently shaking. Protein bound to DNA oligo was washed thrice using a magnetic stand, sedimented, and resuspended in SDS-PAGE loading buffer before denaturation and electrophorectic protein separation by SDS-PAGE followed by Western blot analysis.

Flow cytometry and cell cycle analysis

Cell suspensions and tumor tissues were prepared as described previously (39) and stained with different combinations of fluorophore-coupled antibodies against IFN- $\gamma$ (BD Biosciences), BCMA, CD38, and 
IgG- $\lambda$ (BioLegend). LIVE/DEAD Fixable Aqua Dead Cell Stain was purchased from Invitrogen. Cell Cycle analysis was carried out according to the manufacturer's instructions (BD Biosciences). Fluorescence data were collected on Accuri (BD Accuri C6) and analyzed using FlowJo software (Tree Star).

PCR

Transcript amplification was determined from total RNA purified using RNeasy Kit (QIAGEN). cDNA was synthesized using the iScript cDNA Synthesis Kit (Bio-Rad). RT-PCR was performed in triplicate using the Chromo4 Real-Time Detector (Bio-Rad). The housekeeping genes human TUBB (F, CTTCGGCCAGATCTTCAGAC; R, AGAGAGTGGGTCAGCTGGAA) and murine Gapdh (F, CTGGAGAAACCTGCCAAGTA; R, TGTTGCTGTAGCCGTATTCA) were used as internal controls to normalize target gene mRNA levels. Primers were obtained from RealTimePrimers (human IRF4, F, GTTTAAAGGAAAGTTCCGAGAAGG; R, TACACTTTGTACGGGTCTGAGATG; human $M Y C$, F, CGACGAGACCTTCATCAAAA; R, TGCTGTCGTTGAGAGGGTAG; murine $M y c$, F, GCCCAGTGAGGATATCTGGA; R, ATCGCAGATGAAGCTCTGGT; human PRDM1, F, TGAGAGTGCACAGTGGAGAA; R, ATTGCTGGTGCTGCTAAATC; human TNFAIP3, F, CGTCCAGGTTCCAGAACACCATTC; R, TGCGCTGGCTCGATCTCAGTTG; human CASP3, F, TGGTTCATCCAGTCGCTTTG; R, AATTTCATCCAGTCGCTTTG; human CDKN2A, F, CTTCCCCCACTACCGTAAAT; R, TGCTCACTCCAGAAAACTCC; human CDKN2B, F, CGGAAATCCCCTTATGACTT; R, CACTGAGTTTGCAACAGTGC; human $M M P 1$, F, CATGACTTTCCTGGAATTGG; R, CCTGCAGTTGAACCAGCTAT; human BIRC5, F, TCCCTGGCTCCTCTACTGTT; R, TGTCTCCTCATCCACCTGAA; human TNFRSF17, F, TGACAGTTTGTTGCATGCTT; R, TAAGCTCAGTCCCAAACAGG; human $C D K N 1 B$, F, TCAAACGTGCGAGTGTCTAA; R, CCACTCGTACTTGCCCTCTA; human CDK6, F, TGGAGACCTTCGAGCACC; R, CACTCCAGGCTCTGGAACTT; human BCL2, F, ATGTGTGTGGAGAGCGTCAACC; R, TGAGCAGAGTCTTCAGAGACAGCC; human CCND2, F, ACGGTACTGCTGCAGGCTAT, R, AGCTGCTGGCTAAGATCACC; human GADD45A, F, GCAGGATCCTTCCATTGAGA; R, CTCTTGGAGACCGACGCTG. All primers in 5'-3' orientation.).

\section{ELISA}

Antigen binding affinity of modified antibodies was assessed using the Human IL-6 ELISA Ready-SET-Go! Kit (eBioscience) using recombinant human IRF4 protein purchased from LifeSpan BioSciences. For coating, $2 \mu \mathrm{g} / \mathrm{mL}$ recombinant protein was used and incubated at $4^{\circ} \mathrm{C}$ overnight. To determine the binding affinity, $0.125 \mu \mathrm{g}$ modified antibody was used and incubated for 2 hours at room temperature, and ELISA procedure was carried out according to the manufacturer's instructions. Human $\operatorname{Ig} G \lambda$ chain was detected using the human IgG $\lambda$ light chain ELISA kit according to the manufacturer's instructions (MyBioSource.com).

\section{Statistics}

Statistical analyses were performed using Prism (GraphPad) software. The overall significance for each graph was calculated using 2-tailed Student's $t$ test. $P$ values of less than 0.05 were considered statistically significant.

\section{Study approval}

Mouse care and experimental procedures with mice were performed under pathogen-free conditions in accordance with established institutional guidance and approved protocols from the Research Animal Care Committees of the City of Hope.

\section{Author contributions}

$\mathrm{AH}$ designed the concept of the study, performed the majority of the experiments, interpreted the data, and wrote the manuscript, TN, YJL, and CY performed in vitro studies and analyses after in vivo antibody treatment experiments; CZ performed ex vivo experiments on T cells/IFN- $\gamma$; TN, CL, RM, and YJL assessed antibody and antigen studies by ELISA as well as in vivo efficacy of treatments with modified antibodies; and HY contributed to the concept of the study, designed many experiments, interpreted the data, and wrote the manuscript.

\section{Acknowledgments}

We would like to acknowledge Yanwen Fu of LACell Therapeutics, Sorrento Therapeutics, for developing the DNA oligo-antibody conjugation chemistry used in the studies and for modifying and purifying all the 
antibodies described in the manuscript. We thank the dedicated staff members at the flow cytometry core and light microscopy core at the Beckman Research Institute at City of Hope for their technical assistance. We also acknowledge the contribution of staff members at the animal facilities at City of Hope. This work was supported in part by National Cancer Institute R01CA122976. Research reported in this publication was also supported under grant number P30CA033572. The content is solely the responsibility of the authors and does not necessarily represent the official views of the NIH.

Address correspondence to: Andreas Herrmann, LACell at Sorrento Therapeutics, 4955 Directors Place, San Diego, California 92121, USA. Phone: 858.203.4187; Email: aherrmann@sorrentotherapeutics.com. Or to: Hua Yu, Department of Immuno-Oncology, Beckman Research Institute at City of Hope Comprehensive Cancer Center, Duarte, California 91741, USA. Phone: 626.218.3365; Email: hyu@coh.org.

1. Ferrara N, Adamis AP. Ten years of anti-vascular endothelial growth factor therapy. Nat Rev Drug Discov. 2016;15(6):385-403.

2. Pardoll DM. The blockade of immune checkpoints in cancer immunotherapy. Nat Rev Cancer. 2012;12(4):252-264.

3. Curiel TJ, et al. Blockade of B7-H1 improves myeloid dendritic cell-mediated antitumor immunity. Nat Med. 2003;9(5):562-567.

4. Ferrara N, Hillan KJ, Gerber HP, Novotny W. Discovery and development of bevacizumab, an anti-VEGF antibody for treating cancer. Nat Rev Drug Discov. 2004;3(5):391-400.

5. Leavy O. Therapeutic antibodies: past, present and future. Nat Rev Immunol. 2010;10(5):297.

6. Scott AM, Wolchok JD, Old LJ. Antibody therapy of cancer. Nat Rev Cancer. 2012;12(4):278-287.

7. Peng D, et al. Epigenetic silencing of TH1-type chemokines shapes tumour immunity and immunotherapy. Nature. 2015;527(7577):249-253.

8. Hudis CA. Trastuzumab - mechanism of action and use in clinical practice. N Engl J Med. 2007;357(1):39-51.

9. Gerber HP, Ferrara N. Pharmacology and pharmacodynamics of bevacizumab as monotherapy or in combination with cytotoxic therapy in preclinical studies. Cancer Res. 2005;65(3):671-680.

10. Kaplon H, Reichert JM. Antibodies to watch in 2018. MAbs. 2018;10(2):183-203.

11. Lackey CA, Press OW, Hoffman AS, Stayton PS. A biomimetic $\mathrm{pH}$-responsive polymer directs endosomal release and intracellular delivery of an endocytosed antibody complex. Bioconjug Chem. 2002;13(5):996-1001.

12. Kim A, et al. Intracellular delivery of charge-converted monoclonal antibodies by combinatorial design of block/homo polyion complex micelles. Biomacromolecules. 2016;17(2):446-453.

13. Chen BX, Erlanger BF. Intracellular delivery of monoclonal antibodies. Immunol Lett. 2002;84(1):63-67.

14. Hansen JE, et al. Targeting cancer with a lupus autoantibody. Sci Transl Med. 2012;4(157):157ra142.

15. Noble PW, Young MR, Bernatsky S, Weisbart RH, Hansen JE. A nucleolytic lupus autoantibody is toxic to BRCA2-deficient cancer cells. Sci Rep. 2014;4:5958.

16. Weisbart RH, et al. DNA-dependent targeting of cell nuclei by a lupus autoantibody. Sci Rep. 2015;5:12022.

17. Noble PW, Chan G, Young MR, Weisbart RH, Hansen JE. Optimizing a lupus autoantibody for targeted cancer therapy. Cancer Res. 2015;75(11):2285-2291.

18. Guo K, et al. Targeting intracellular oncoproteins with antibody therapy or vaccination. Sci Transl Med. 2011;3(99):99ra85.

19. Shin SM, et al. Antibody targeting intracellular oncogenic Ras mutants exerts anti-tumour effects after systemic administration. Nat Commun. 2017;8:15090.

20. Yu H, Lee H, Herrmann A, Buettner R, Jove R. Revisiting STAT3 signalling in cancer: new and unexpected biological functions. Nat Rev Cancer. 2014;14(11):736-746.

21. Yu H, Pardoll D, Jove R. STATs in cancer inflammation and immunity: a leading role for STAT3. Nat Rev Cancer. 2009;9(11):798-809.

22. Yu H, Kortylewski M, Pardoll D. Crosstalk between cancer and immune cells: role of STAT3 in the tumour microenvironment. Nat Rev Immunol. 2007;7(1):41-51.

23. Yu H, Jove R. The STATs of cancer — new molecular targets come of age. Nat Rev Cancer. 2004;4(2):97-105.

24. Wang S, et al. Cellular uptake mediated by epidermal growth factor receptor facilitates the intracellular activity of phosphorothioate-modified antisense oligonucleotides. Nucleic Acids Res. 2018;46(7):3579-3594.

25. Krieg AM. Therapeutic potential of Toll-like receptor 9 activation. Nat Rev Drug Discov. 2006;5(6):471-484.

26. Kortylewski M, et al. In vivo delivery of siRNA to immune cells by conjugation to a TLR9 agonist enhances antitumor immune responses. Nat Biotechnol. 2009;27(10):925-932.

27. Sachs HG, Stambrook PJ, Ebert JD. Changes in membrane potential during the cell cycle. Exp Cell Res. 1974;83(2):362-366.

28. Huang X, et al. Prolonged early G(1) arrest by selective CDK4/CDK6 inhibition sensitizes myeloma cells to cytotoxic killing through cell cycle-coupled loss of IRF4. Blood. 2012;120(5):1095-1106.

29. Shaffer AL, et al. IRF4 addiction in multiple myeloma. Nature. 2008;454(7201):226-231.

30. Shaffer AL, Emre NC, Romesser PB, Staudt LM. IRF4: immunity. Malignancy! Therapy? Clin Cancer Res. 2009;15(9):2954-2961.

31. Kessler JD, et al. A SUMOylation-dependent transcriptional subprogram is required for Myc-driven tumorigenesis. Science. 2012;335(6066):348-353.

32. Zhu YX, et al. Cereblon expression is required for the antimyeloma activity of lenalidomide and pomalidomide. Blood. 2011;118(18):4771-4779.

33. Yang Y, et al. Exploiting synthetic lethality for the therapy of ABC diffuse large B cell lymphoma. Cancer Cell. 2012;21(6):723-737.

34. Yeatman TJ. A renaissance for SRC. Nat Rev Cancer. 2004;4(6):470-480.

35. Warmuth M, Damoiseaux R, Liu Y, Fabbro D, Gray N. SRC family kinases: potential targets for the treatment of human cancer and leukemia. Curr Pharm Des. 2003;9(25):2043-2059. 
36. Irby RB, Yeatman TJ. Role of Src expression and activation in human cancer. Oncogene. 2000;19(49):5636-5642.

37. Niu G, et al. Roles of activated Src and Stat3 signaling in melanoma tumor cell growth. Oncogene. 2002;21(46):7001-7010.

38. Liu L, et al. A novel 7-bromoindirubin with potent anticancer activity suppresses survival of human melanoma cells associated with inhibition of STAT3 and Akt signaling. Cancer Biol Ther. 2012;13(13):1255-1261.

39. Buettner R, Mesa T, Vultur A, Lee F, Jove R. Inhibition of Src family kinases with dasatinib blocks migration and invasion of human melanoma cells. Mol Cancer Res. 2008;6(11):1766-1774.

40. Niu G, et al. Role of Stat3 in regulating p53 expression and function. Mol Cell Biol. 2005;25(17):7432-7440

41. Frey S, Richter RP, Görlich D. FG-rich repeats of nuclear pore proteins form a three-dimensional meshwork with hydrogel-like properties. Science. 2006;314(5800):815-817.

42. Pensado A, Fernandez-Piñeiro I, Seijo B, Sanchez A. Anionic nanoparticles based on Span 80 as low-cost, simple and efficient non-viral gene-transfection systems. Int J Pharm. 2014;476(1-2):23-30.

43. Wilhelm C, Billotey C, Roger J, Pons JN, Bacri JC, Gazeau F. Intracellular uptake of anionic superparamagnetic nanoparticles as a function of their surface coating. Biomaterials. 2003;24(6):1001-1011.

44. Wolpoe ME, et al. HER-2/neu-specific monoclonal antibodies collaborate with HER-2/neu-targeted granulocyte macrophage colony-stimulating factor secreting whole cell vaccination to augment CD8+ T cell effector function and tumor-free survival in Her-2/neu-transgenic mice. J Immunol. 2003;171(4):2161-2169.

45. Duraiswamy J, Freeman GJ, Coukos G. Therapeutic PD-1 pathway blockade augments with other modalities of immunotherapy T-cell function to prevent immune decline in ovarian cancer. Cancer Res. 2013;73(23):6900-6912.

46. Herrmann A, et al. CTLA4 aptamer delivers STAT3 siRNA to tumor-associated and malignant T cells. J Clin Invest. 2014;124(7):2977-2987. 